\title{
ApoE4 disrupts interaction of sortilin with fatty acid-binding protein 7 essential to promote lipid signaling
}

\author{
Antonino Asaro ${ }^{1, *}$, Rishabhdev Sinha ${ }^{1, *}$, Magda Bakun², Oleksandra Kalnytska ${ }^{1}$, \\ Anne-Sophie Carlo-Spiewok ${ }^{1}$, Tymon Rubel ${ }^{3}$, Annemieke Rozeboom ${ }^{4,5}$, Michal Dadlez ${ }^{2,6}$, Bozena Kaminska ${ }^{7}$, \\ Eleonora Aronica ${ }^{4}$, Anna R. Malik ${ }^{1,7}$ and Thomas E. Willnow ${ }^{1,8, \pm}$
}

\begin{abstract}
Sortilin is a neuronal receptor for apolipoprotein E (apoE). Sortilindependent uptake of lipidated apoE promotes conversion of polyunsaturated fatty acids (PUFA) into neuromodulators that induce anti-inflammatory gene expression in the brain. This neuroprotective pathway works with the apoE3 variant but is lost with the apoE4 variant, the main risk factor for Alzheimer's disease (AD). Here, we elucidated steps in cellular handling of lipids through sortilin, and why they are disrupted by apoE4. Combining unbiased proteome screens with analyses in mouse models, we uncover interaction of sortilin with fatty acid-binding protein 7 (FABP7), the intracellular carrier for PUFA in the brain. In the presence of apoE3, sortilin promotes functional expression of FABP7 and its ability to elicit lipid-dependent gene transcription. By contrast, apoE4 binding blocks sortilin-mediated sorting, causing catabolism of FABP7 and impairing lipid signaling. Reduced FABP7 levels in the brain of AD patients expressing apoE4 substantiate the relevance of these interactions for neuronal lipid homeostasis. Taken together, we document interaction of sortilin with mediators of extracellular and intracellular lipid transport that provides a mechanistic explanation for loss of a neuroprotective lipid metabolism in AD.
\end{abstract}

KEY WORDS: Alzheimer's disease, Fatty acid binding protein, Polyunsaturated fatty acid, Protein sorting, VPS10P domain receptor

\section{INTRODUCTION}

Apolipoprotein E (apoE) is the main carrier for lipids in the brain. It is released by astrocytes and microglia and delivers essential lipids to neurons that take up apoE-bound cargo through apoE receptors expressed on the neuronal cell surface (reviewed in Holtzman et al.,

\footnotetext{
${ }^{1}$ Max-Delbrueck-Center for Molecular Medicine, 13125 Berlin, Germany. ${ }^{2}$ Mass Spectrometry Laboratory, Institute of Biochemistry and Biophysics, Polish Academy of Sciences, 02-106 Warsaw, Poland. ${ }^{3}$ Warsaw University of Technology, Institute of Radioelectronics and Multimedia Technology, 00-665 Warsaw, Poland. ${ }^{4}$ Department of (Neuro) Pathology, Amsterdam UMC, University of Amsterdam, Amsterdam Neuroscience, 1105AZ Amsterdam, The Netherlands. ${ }^{5}$ Center for Neuroscience, Amsterdam Institute for Life Sciences, University of Amsterdam, 1098XH Amsterdam, The Netherlands. ${ }^{6}$ Biology Department, Institute of Genetics and Biotechnology 02-106 Warsaw, Poland. ${ }^{7}$ Nencki Institute of Experimental Biology, 02-093 Warsaw, Poland. ${ }^{8}$ Department of Medical Biochemistry, Aarhus University, 8000 Aarhus, Denmark.

*These authors contributed equally to this work

Author for correspondence (willnow@mdc-berlin.de)
}

(D.A., 0000-0001-9610-5381; T.E.W., 0000-0001-9515-7921

This is an Open Access article distributed under the terms of the Creative Commons Attribution License (https://creativecommons.org/licenses/by/4.0), which permits unrestricted use,

distribution and reproduction in any medium provided that the original work is properly attributed.

Handling Editor: Giampietro Schiavo

Received 10 May 2021; Accepted 16 September 2021
2012). ApoE also bears significance as the most important genetic risk factor for the sporadic form of Alzheimer's disease (AD) as carriers of the $A P O E \varepsilon 4$ allele (encoding apoE4) are at a significantly higher risk of developing $\mathrm{AD}$ than individuals having the common $A P O E \varepsilon 3$ gene variant (encoding apoE3) (Corder et al., 1993).

The identification of sortilin as a receptor for neuronal clearance of apoE has shed some light on cellular mechanisms implicating this apolipoprotein in brain lipid metabolism and AD progression (Carlo et al., 2013). In detail, sortilin directs neuronal uptake and conversion of apoE-bound $\omega 3$-polyunsaturated fatty acids (PUFA) into endocannabinoids (eCBs), neuromodulatory lipids that act via nuclear receptors of the peroxisome proliferator-activated receptor (PPAR) family to induce an anti-inflammatory gene expression profile in the brain (Asaro et al., 2020). This neuroprotective action of sortilin is seen with apoE3 but lost when binding apoE4, disrupting neuronal eCB production and resulting in a proinflammatory state that may predispose the apoE4 brain to neurodegeneration (Asaro et al., 2020). However, the molecular mechanism of action for sortilin in neuronal lipid homeostasis and why this activity is lost in the presence of apoE4 remains poorly understood.

Sortilin is a member of the vacuolar protein sorting 10 protein (VPS10P) domain receptor family, a group of specialized receptors involved in endocytosis and intracellular sorting of ligands (reviewed in Carlo et al., 2014). Binding of lipid-laden apoE4 to sortilin on the cell surface does not block internalization but disrupts succeeding steps in intracellular receptor sorting (Asaro et al., 2020; Carlo et al., 2013). This observation suggests that it is not the delivery of PUFA into neurons but subsequent steps in intracellular handling of the lipids that are disrupted in the presence of apoE4. Here, we aimed at dissecting the distinct steps of sortilin action in neuronal eCB metabolism impaired by apoE4. Combining unbiased proteome screens in primary neurons and glia with studies in mouse models and AD patient specimens, we uncovered the functional interaction of sortilin with fatty acid-binding protein 7 (FABP7), the intracellular carrier for PUFA and eCBs in the brain (Balendiran et al., 2000; Kaczocha et al., 2009; Xu et al., 1996). ApoE4 disrupts the ability of sortilin to promote stability and proper intracellular sorting of FABP7, which is essential for lipid signaling via PPARs. These findings identified a central role for sortilin in neuroprotective lipid metabolism by integrating extracellular (apoE) and intracellular (FABP7) lipid transport processes that are disrupted by apoE4-induced missorting of this receptor.

\section{RESULTS}

Surface proteome analyses identify FABP7 as novel sortilin target in neurons

Sortilin is a sorting receptor that directs proteins between the cell surface and various intracellular compartments. To identify 
sortilin-dependent trafficking processes relevant for neuronal lipid metabolism and action, we applied an unbiased proteomics approach to identify novel receptor targets in neurons. This approach was based on our assumption that the loss of sortilin activity will result in aberrant distribution of so-far-unknown receptor ligands between cell surface and intracellular compartments. Such unbiased screens have been used successfully by us before to identify targets for related VPS10P domain receptors (Malik et al., 2019; Subkhangulova et al., 2018).

To identify sortilin ligands, we biotinylated surface proteins in primary neurons from wild-type (WT) mice or animals genetically deficient for Sort1, the gene encoding sortilin (KO) (Jansen et al., 2007). The biotinylated proteins were purified from cell extracts using streptavidin beads, identified by liquid chromatography tandem mass spectrometry (LC-MS/MS), and subjected to LCMS label-free quantification (workflow in Fig. 1A). By this approach, we identified multiple proteins with altered abundance in the neuronal cell surface fraction upon loss of sortilin (Fig. 1B; Tables S1 and S4); some of which may be primary, others secondary targets of receptor dysfunction in $\mathrm{KO}$ neurons. Proteins with altered abundance in the cell surface fraction of KO neurons included known receptor ligands, such as tropomyosin receptor kinase B (TrkB) (Vaegter et al., 2011), EGF receptor (Al-Akhrass et al., 2017), sphingomyelin phosphodiesterase (Ni and Morales, 2006) and vacuolar protein sorting-associated protein 26B (Kim et al., 2010) (Fig. 1B; Table S1). These findings corroborated our strategy for identifying primary targets of sortilin-mediated protein sorting. Among the proteins with altered surface abundance in $\mathrm{KO}$ neurons was one hit with particular relevance to PUFA and $\mathrm{eCB}$ metabolism, namely brain fatty acid binding protein 7 (FABP 7). The abundance of FABP7 was increased in the surface proteome of $\mathrm{KO}$ neurons, suggesting altered subcellular distribution of the carrier in the absence of sortilin (Fig. 1B; Table S1). This effect was specific to FABP7 and not seen for other fatty acid-binding proteins expressed in the brain, namely FABP3 and FABP5 (Table S1).

To query the specificity of sortilin-dependent sorting of FABP7 in neurons, we repeated the quantitative surface proteome analysis in primary astrocytes from WT and KO mice (Fig. 1C; Tables S1 and S5). Again, established sortilin interaction partners were identified as being altered in their presence in the cell surface fraction, including TrkB, amyloid-like protein 2 (Butkinaree et al.,
2015), ATP-binding cassette transporter ABCA1 (Lv et al., 2019) and progranulin (Hu et al., 2010). By contrast, cell surface localization of FABP7 was not impacted by sortilin deficiency in astrocytes (Fig. 1C; Table S1).

\section{Sortilin facilitates expression of FABP7 in CHO cells}

FABP7 is a brain-specific lipid chaperone that enables cellular uptake and cytosolic trafficking of PUFA and eCBs (Feng et al., 1994; Kaczocha et al., 2009; Kurtz et al., 1994). FABP7-dependent trafficking is required for proper biosynthesis of eCBs from PUFA, and for their delivery to nuclear PPARs for regulation of gene transcription (reviewed in Moulle et al., 2012). When Myc-tagged FABP7 and sortilin were co-expressed in Chinese hamster ovary cells $(\mathrm{CHO}-\mathrm{S} / \mathrm{F})$, sortilin co-immunoprecipitated with an affinity resin directed against the Myc epitope in FABP7, confirming FABP7 as sortilin ligand (Fig. 2A). Co-immunoprecipitation was also seen in the presence of apoE3 or apoE4, arguing that FABP7 and apoE simultaneously bind to their receptor sortilin (Fig. 2B). Interaction of sortilin with FABP7 was supported by proximity ligation assay (PLA) results, documenting close proximity of both proteins in intracellular compartments of $\mathrm{CHO}-\mathrm{S} / \mathrm{F}$ cells (Fig. 2C). In subcellular fractionation experiments, FABP7 and sortilin mainly colocalized in the trans-Golgi network (TGN), early endosomes and the cell surface fraction of CHO-S/F cells (Fig. 2D), consistent with a role for sortilin in sorting of cargo between plasma membrane and secretory and endocytic compartments (Nielsen et al., 2001).

Of note, levels of FABP7 were significantly higher in $\mathrm{CHO}$ cells expressing sortilin (CHO-S) as compared with parental $\mathrm{CHO}$ cells lacking the receptor (Fig. 3A,B). This effect was specific for FABP7 as levels of the co-transfected green fluorescent protein (GFP) were identical in both cell lines (Fig. 3A). Elevated levels of FABP7 in $\mathrm{CHO}-\mathrm{S}$ as compared with parental $\mathrm{CHO}$ cells were due to an increase in FABP7 half-life as shown by comparing the turnover of the protein in $\mathrm{CHO}$ and $\mathrm{CHO}-\mathrm{S}$ cells following treatment with cycloheximide, an inhibitor of protein translation (Fig. 3C,D).

\section{Sortilin promotes neuronal expression of FABP7 in an apoE isoform-dependent manner}

So far, our studies documented a direct interaction of sortilin with FABP7 that controlled stability and intracellular distribution of the
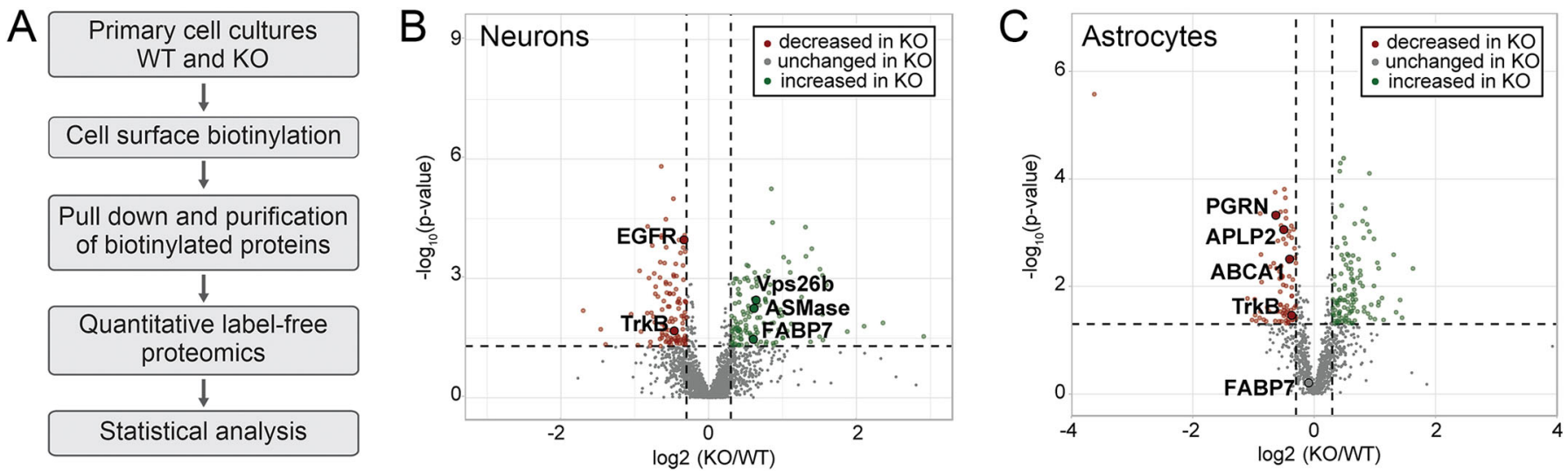

Fig. 1. Surface proteome analysis identifies fatty acid-binding protein 7 as novel sortilin target in neurons but not astrocytes. (A) Workflow of surface proteome analysis in primary neurons from wild-type (WT) and Sort1 ${ }^{-1-}(\mathrm{KO})$ mice. (B) Comparison of the surface proteomes of WT and KO primary neurons ( $n=3$ biological replicates/group; two technical replicates per biological replicate). Dashed lines show threshold values for log2 (fold change) and -log10 ( $P$-value), \pm 0.3 and 1.3, respectively. Proteins with increased (green) or decreased (red) levels in the surface proteome of KO neurons are color-coded. Selected proteins with altered surface exposure in KO neurons are highlighted (see Table S1 for details). (C) Data as in B but comparing the surface proteomes of WT and KO primary astrocytes ( $n=6$ biological replicates/group). Selected proteins with altered surface exposure in KO astrocytes are highlighted (see Table $\mathrm{S} 1$ for details) 

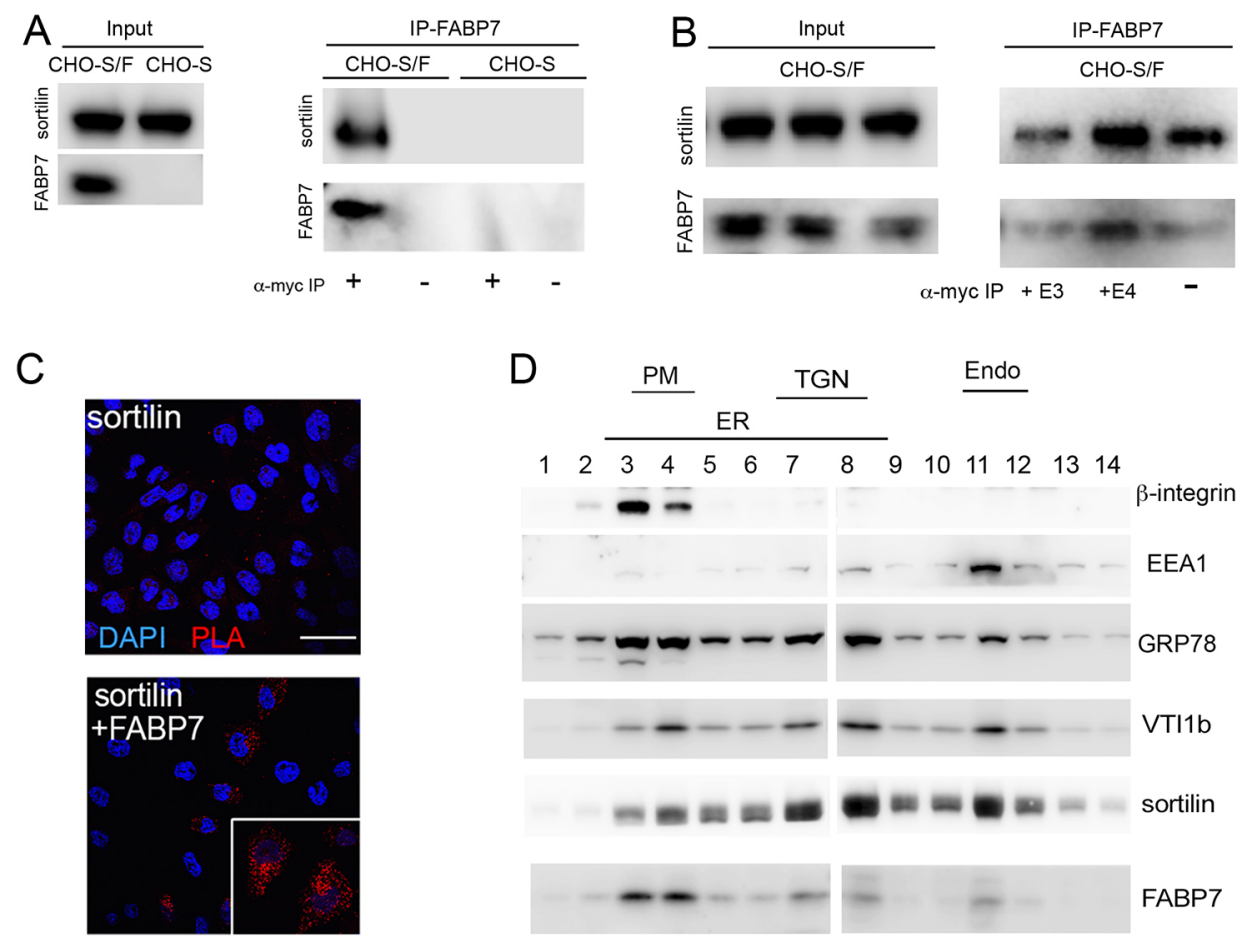

Fig. 2. Sortilin and FABP7 interact in cells. (A) Chinese hamster ovary $(\mathrm{CHO})$ cells stably overexpressing sortilin (CHO-S) or sortilin and FABP7 tagged with a Myc epitope (CHO-S/F) were used for co-immunoprecipitation (IP) experiments. Expression of sortilin and FABP7-Myc in total cell lysates of both cell lines is shown in the panel Input (15 $\mu \mathrm{g}$ of total cell lysate). In panel IP-FABP7, co-immunoprecipitation of sortilin with anti-Myc affinity resin (+) is seen in lysates from $\mathrm{CHO}-\mathrm{S} / \mathrm{F}$ but not from CHO-S cells. No immunoprecipitation of sortilin with anti-Myc affinity resin is seen in CHO-S cells, or in $\mathrm{CHO}-\mathrm{S} / \mathrm{F}$ in the absence of the affinity resin (-). (B) Co-immunoprecipitation of sortilin with FABP7-myc from CHO-S/F cells as described in A. Prior to immunoprecipitation with anti-Myc affinity resin, cells were treated with conditioned medium from HEK293 cells containing $5 \mu \mathrm{g} / \mathrm{ml}$ of apoE3 (+E3) or apoE4 (+E4; see Materials and Methods for details) or blank medium (-) for $24 \mathrm{~h}$. (C) Proximity ligation assay (PLA) to assess close spatial proximity of sortilin and FABP7 in CHO cells. Primary antibodies were directed against sortilin or the Myc epitope in FABP7-Myc. Close proximity is detected in CHO cells expressing both sortilin and FABP7 (red signal). No PLA signal is seen in cells expressing sortilin only. Cell nuclei were counterstained with DAPI (blue). The inset shows a higher magnification image of cells positive for PLA signals for sortilin and FABP7-Myc. Scale bar: $50 \mu \mathrm{m}$. (D) Subcellular fractionation of CHO-S/F cells using gradient ultracentrifugation. Fractions were identified based on markers for endoplasmic reticulum (ER; GRP78), plasma membrane (PM; $\beta$-integrin), trans-Golgi network (TGN; VTI1b), and early endosomes (Endo; EEA1). FABP7 colocalizes with sortilin to the PM (fractions 3-4), TGN (fractions 7-8), and endosomes (fraction 11). Images shown are representative of results from three independent experiments.

lipid carrier in CHO cells (Fig. 3) and neurons (Fig. 1B). To test whether this interaction may also be relevant for control of FABP7 levels in vivo, we studied levels of the carrier in brains of WT and $\mathrm{KO}$ mice. Given the importance of apoE genotype for sortilin action in brain lipid homeostasis (Asaro et al., 2020), WT and KO mice carried a targeted replacement of the murine Apoe locus with genes encoding human apoE3 (E3) or apoE4 (E4) (Knouff et al., 1999). In the presence of apoE3, total brain levels of FABP7 were significantly higher in WT mice as compared to animals lacking sortilin (E3/WT versus E3/KO; Fig. 4A,C). However, in apoE4 mice, sortilin did not impact FABP7 expression, as levels of the carrier were comparable in E4/WT and E4/KO animals (Fig. 4B,C). As a control, sortilin deficiency did not affect levels of FABP5 in E3 or E4 animals (Fig. 4A,B). The beneficial effect of sortilin activity on FABP7 levels in E3 mice, but not in E4 animals, manifested through a post-transcriptional mechanism as brain levels of Fabp7 transcripts were not altered comparing E3 and E4 animals, either WT or KO for Sort1 (Fig. 4D). These experiments substantiate the importance of sortilin for promoting FABP7 levels in the brain of mice expressing apoE3, but not apoE4. An effect of apoE isoform on FABP7 levels was also seen in the human AD brain as levels of the protein were significantly higher in patients of the APOE 3 / $A P O E \varepsilon 3$ as compared with the APOE\&4/APOE 4 genotype (Fig. 4E,F; Table S2). As in the mouse brain, levels of FABP5 were comparable in both genotypes (Fig. 4E).
In the adult mouse brain, predominant expression of FABP7 has been localized to several types of glia, such as ependyma and Bergmann glia (Kurtz et al., 1994). However, using immunohistology we also detected moderate but consistent coexpression of FABP7 with sortilin in cortical neurons marked by NeuN (Fig. 5A). Neuronal expression of FABP7 was substantiated in human $\mathrm{AD}$ brain tissue (Fig. 5B; Table S3). To document that decreased levels of FABP7 seen in apoE3 mice upon deletion of sortilin $(\mathrm{E} 3 / \mathrm{KO})$ represented loss from neurons normally coexpressing both proteins, we performed comparative expression analyses in primary neurons and astrocytes from all four genotypes. As in the brain, co-expression of FABP7 with sortilin was seen in cultured WT neurons (Fig. 6A). These cell cultures produced apoE3 and apoE4, likely from astrocytes present in these neuron-enriched cultures (Fig. 6B). The relevance of sortilin and apoE3 interaction for control of neuronal levels of FABP7 was substantiated by western blot analysis documenting significantly higher levels of FABP7 in E3/WT as compared to E3/KO neurons (Fig. 6C,D). This beneficial effect of sortilin on FABP7 was lost with apoE4, as levels of the carrier were significantly lower in E4/WT as compared to E3/ WT neurons (Fig. 6C,D). As in the total brain, loss of sortilin activity did not impact levels of FABP5 in either apoE genotype (Fig. 6C). Also, levels of sortilin were not changed comparing E3/ WT and E4/WT neurons (Fig. 6E). The difference in FABP7 levels comparing E3/WT and E3/KO cell preparations was not due to a 


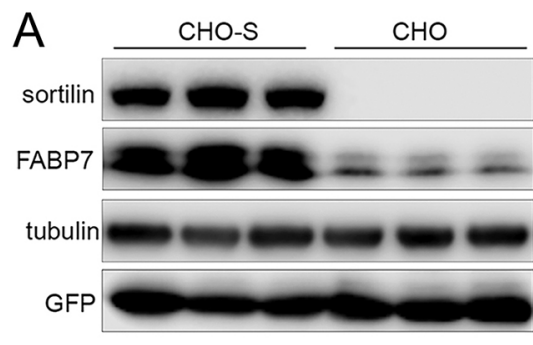

$\mathrm{B}$

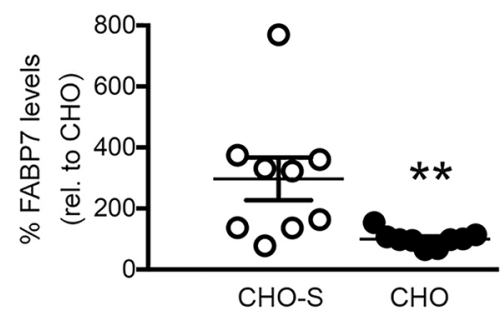

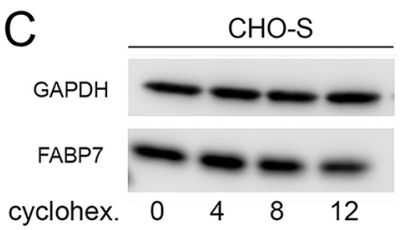
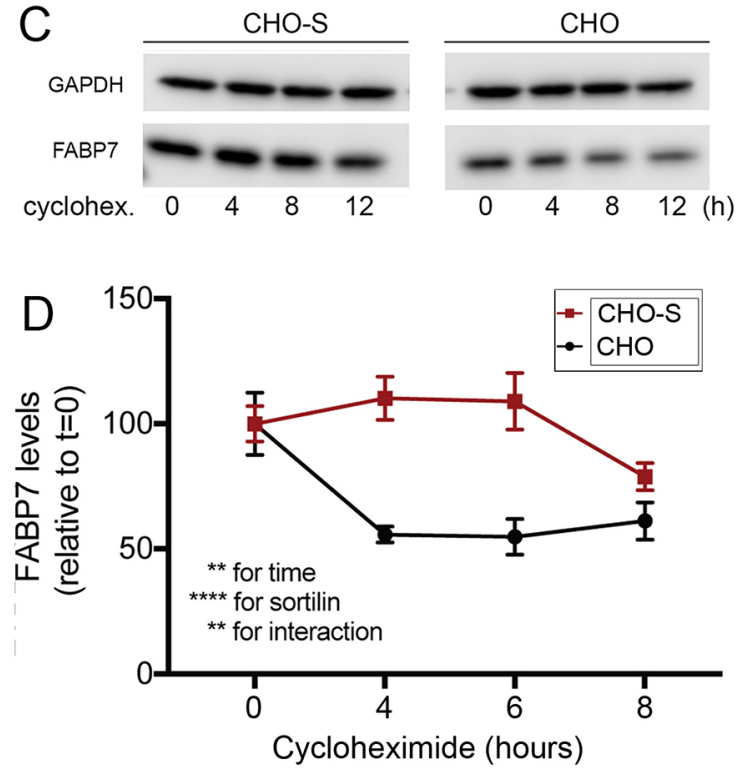

Fig. 3. Sortilin stabilizes cellular levels of FABP7 in $\mathbf{C H O}$ cells. (A,B) Parental Chinese hamster ovary (CHO) cells or $\mathrm{CHO}$ cells stably expressing sortilin (CHO-S) were transiently transfected with expression constructs encoding for FABP7 and GFP. A representative western blot of documenting expression of sortilin and FABP7 in replicate lysates of $\mathrm{CHO}$ and $\mathrm{CHO}-\mathrm{S}$ cells is shown in A. Detection of GFP and tubulin served as transfection and loading controls, respectively. $\mathrm{B}$ shows $\mathrm{FABP} 7$ levels in $\mathrm{CHO}$ and $\mathrm{CHO}-\mathrm{S}$ transfectants as determined by densitometric scanning of replicate western blots $(n=9$ replicates from 3 independent experiments per cell line). Data are mean \pm s.e.m. given as percentage of FABP7 levels in CHO cells (set to $100 \%$ ). Levels of FABP7 (but not of GFP) are significantly increased by the presence of sortilin in $\mathrm{CHO}-\mathrm{S}$ compared with $\mathrm{CHO}$ cells. ${ }^{* *} P<0.01$ (unpaired, two-tailed Student's $t$-test). (C,D) $\mathrm{CHO}$ and $\mathrm{CHO}-\mathrm{S}$

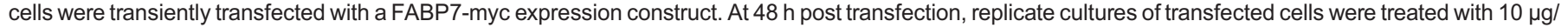

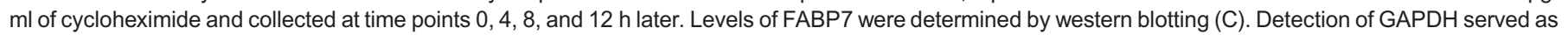

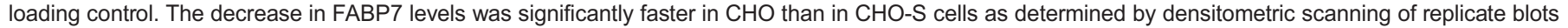

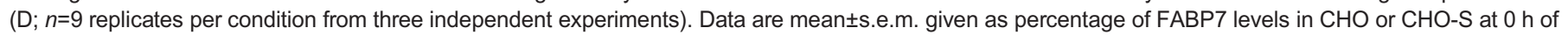
treatment (set to $100 \%$ ). ${ }^{* *} P<0.01$; ${ }^{* * *} P<0.0001$ (two-way ANOVA, followed by Bonferroni post-hoc analysis).

difference in the amounts of FABP7-producing astrocytes in the cultures, as transcript levels of the astrocyte marker Gfap were comparable in both cell preparations (Fig. 6F). Also, levels of Fabp 7 transcript in primary neurons were not impacted by $A P O E$ or Sort1 genotypes, substantiating a post-transcriptional mechanism of expression control (Fig. 6G).

Robust expression of FABP7 was also detected in cultured primary astrocytes from E3/WT and E3/KO mice (Fig. S1A). However, contrary to the situation in neurons, levels of FABP7 protein (Fig. S1B,C) or Fabp7 transcript (Fig. S1E) were not impacted by apoE or sortilin genotypes Also, levels of sortilin were comparable in astrocytes expressing apoE3 or apoE4 (Fig. S1B,D).

Taken together, these findings documented that expression of FABP7 is controlled by sortilin, an activity specific to neurons and not seen in astrocytes. This protective activity is obviously lost in E3 mice lacking sortilin $(\mathrm{E} 3 / \mathrm{KO})$. However, protection of FABP7 is also lost in WT neurons in the presence of apoE4 (E4/WT) as this apoE variant disrupts sortilin trafficking and renders cell essentially deficient for the receptor activity (Asaro et al., 2020).

\section{ApoE4 disrupts the ability of sortilin to control functional expression of FABP7}

So far, our findings have uncovered a role for sortilin in controlling neuronal stability and subcellular localization of FABP7. The ability of sortilin to sustain FABP7 levels in the murine brain, and in cultured neurons thereof, was seen in the presence of apoE3 but not with apoE4. To explore the underlying molecular mechanisms, we applied the $\mathrm{CHO}-\mathrm{S} / \mathrm{F}$ cell model to study the functional interaction of sortilin with FABP7 in the presence of apoE3 or apoE4 (Fig. 7). As in the murine brain, the ability of sortilin to promote FABP7 levels in CHO cells was seen with apoE3 but was compromised by the presence of apoE4. This effect was documented by treating CHO-S/F cells with conditioned medium from HEK293 cells secreting human apoE3 or apoE4 (Chen et al., 2010). When applying identical amounts of both apoE isoforms to the cell medium, levels of FABP7 in $\mathrm{CHO}-\mathrm{S} / \mathrm{F}$ cells were significantly lowered by the addition of apoE4 as compared with apoE3 (Fig. 7A, B). The same effect was seen using native apoE3 or apoE4 secreted by primary astrocytes (Fig. 7C,D).

Because apoE4 disrupts intracellular routing of sortilin (Asaro et al., 2020), we tested whether the ability of the receptor to direct sorting of FABP7 might be compromised by binding of apoE4. To verify this notion, we compared the subcellular distribution of sortilin-FABP7 complexes in CHO-S/F cells treated with apoE3 or apoE4. As shown in Fig. 7E, the intracellular distribution of sortilinFABP7 complexes (as detected through a PLA) changed from a dispersed vesicular pattern with apoE3 to a confined perinuclear appearance with apoE4. A similar effect of apoE4 was seen on sortilin alone but not on FABP7 alone (Fig. 7E). These findings corroborate that apoE4 primarily acts on sortilin, impacting sorting of the fraction of FABP7 bound by this receptor. Altered sorting of sortilin-FABP7 complexes in the presence of apoE4 was confirmed quantitatively by colocalization studies documenting trapping of PLA signals for sortilin and FABP7 in early endosomes (marked by Rab5; Fig. 7F,H) and recycling endosomes (marked by Rab11; Fig. 7G,I) in the presence of apoE4 as compared with apoE3.

Among other functions, FABP7 directs PUFA and eCBs to the nucleus to activate transcription factors of the PPAR family (Adida and Spener, 2006; Mita et al., 2010; Tripathi et al., 2017). To explore whether apoE4-induced missorting of sortilin disrupts 

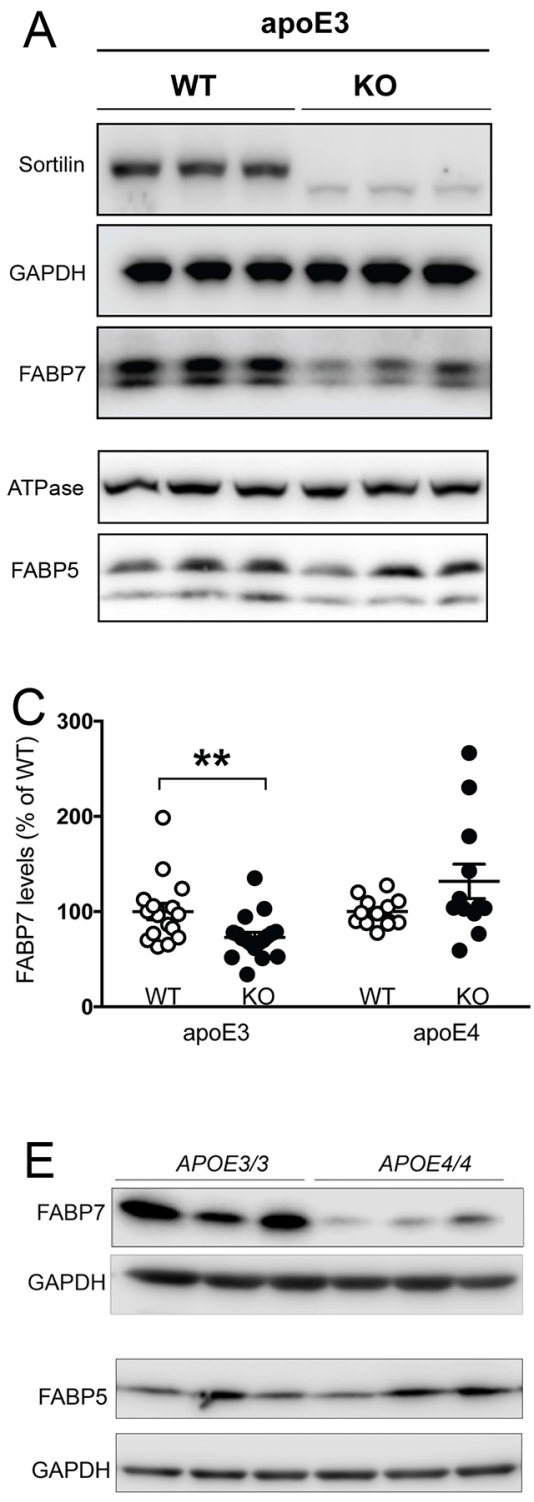

B
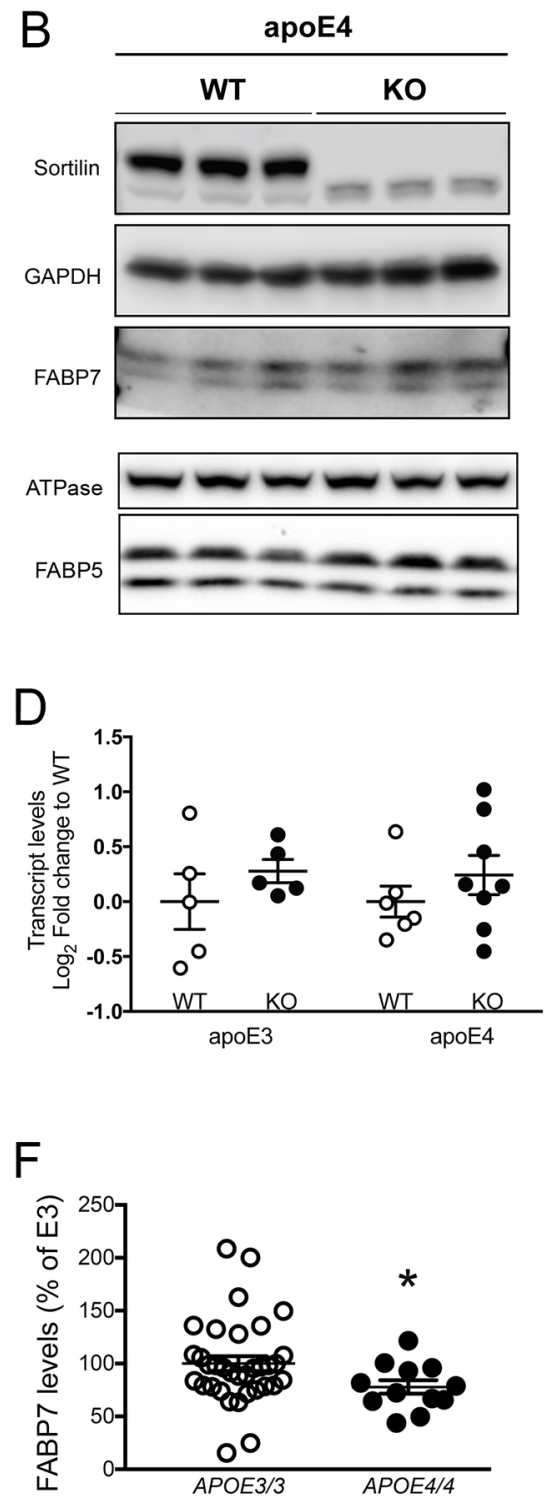

Fig. 4. Sortilin deficiency decreases FABP7 levels in brains of apoE3 but not apoE4 mice. $(A, B)$ Western blot analysis of FABP5 and FABP7 levels in brain cortices of apoE3 (A) and apoE4 (B) targeted replacement mice, either wild-type (WT) or homozygous for the Sort1 null allele (KO) (at 3 months of age). Na/K ATPase and GAPDH served as loading controls for detection of FABP5 and FABP7, respectively. Detection of sortilin served as genotype control. (C) Quantitative analysis of FABP7 levels in brain cortices of apoE3and apoE4-expressing mice of the indicated Sort1 genotype using densitometric scanning of replicate western blots. Values are mean \pm s.e.m. given as percentage of the respective WT control (set to $100 \%$ ); $n=16$ for E3/WT, $n=18$ for E3/KO, $n=12$ for E4 groups. ${ }^{* *} P<0.01$ between genotypes (unpaired, two-tailed Student $t$-test). (D) Levels of Fabp7 transcripts in brain extracts (cortex and hippocampus) of apoE3- and apoE4-expressing mice of the indicated Sort1 genotypes as determined by quantitative RT-PCR. Values are given as mean \pm s.e.m. ( $n=5$ for E3 groups, $n=6$ for E4/ WT, $n=8$ for E4/KO). No statistically significant differences in transcript levels were observed using unpaired, two-tailed Student's $t$-test. (E,F) Western blot analysis of FABP5 and FABP7 levels in prefrontal cortex specimens of $A D$ patients homozygous for $A P O E \varepsilon 3$ or $A P O E \varepsilon 4$ ( pathological characteristics given in Table S2). A representative western blot is shown in E. Detection of GAPDH served as loading control. $F$ shows the result of densitometric scanning of replicate blots. Values are mean \pm s.e.m. given as percentage of $A P O E \varepsilon 3 /$ 3 genotype (mean value set to $100 \%$ ); $n=12-34$ individuals per group. ${ }^{*} P<0.05$ (Welch's $t$-test). this FABP7 function, we transfected $\mathrm{CHO}$ cells with expression constructs encoding a PPAR-inducible firefly luciferase gene as well as a constitutively expressed Renilla luciferase gene (transfection control). Transfection with these reporter constructs conferred PPAR-dependent expression of firefly luciferase to $\mathrm{CHO}$ cells as shown by the increase in firefly luciferase activity achieved upon addition of the PAPR $\gamma$ agonist rosiglitazone to transfectants (Fig. 8A). Co-expression of sortilin and FABP7 in CHO-S/F cells resulted in a significantly higher relative activity of firefly luciferase as compared to $\mathrm{CHO}$ cells expressing either sortilin (CHO-S) or FABP7 (CHO-F) alone (Fig. 8B). PPAR-induced transcription of the firefly luciferase reporter in $\mathrm{CHO}-\mathrm{S} / \mathrm{F}$ cells was reduced by addition of exogenous apoE4 as compared with apoE3, substantiating the detrimental effects of apoE4 on the sortilindependent gene regulatory actions of FABP7 (Fig. 8C).

\section{DISCUSSION}

We propose a novel concept defining a role for sortilin in neuronal lipid metabolism (Fig. 8D,E). By binding of lipidated apoE from the extracellular space, sortilin enables neuronal uptake of essential lipids, including PUFA, the precursors to eCBs. Simultaneous binding of FABP7 to the cytoplasmic tail of the receptor facilitates spatial proximity of apoE and FABP7 in the early endocytic compartments and facilitates lipid transfer from the extracellular to the intracellular transport particle. In the presence of apoE3, proper recycling of sortilin and apoE3 to the cell surface releases lipidladen FABP7, which directs further steps in intracellular metabolism and action of PUFA (Fig. 8D). By contrast, the known propensity of apoE4 to block recycling of sortilin (Asaro et al., 2020) impairs release of FABP7 and blocks subsequent steps in intracellular lipid handling and action (Fig. 8E).

FABP7 is a member of a class of chaperones that facilitate intracellular transport of lipids by increasing their solubility and rate of diffusion in the aqueous milieu of the cytosol (reviewed in Matsumata et al., 2016; Moulle et al., 2012). FABP7 shows highest affinity for docosahexaenoic acid (DHA), the most abundant $\omega 3$ PUFA in the brain, but also binds other fatty acid derivatives, including eCBs (Balendiran et al., 2000; Kaczocha et al., 2009; Xu et al., 1996). FABP7 directs cellular uptake (Kaczocha et al., 2009) and intracellular metabolism (Elmes et al., 2015) of fatty acids. In addition, it facilitates nuclear targeting of DHA and eCBs for activation of PPARs (Adida and Spener, 2006; Mita et al., 2010; 

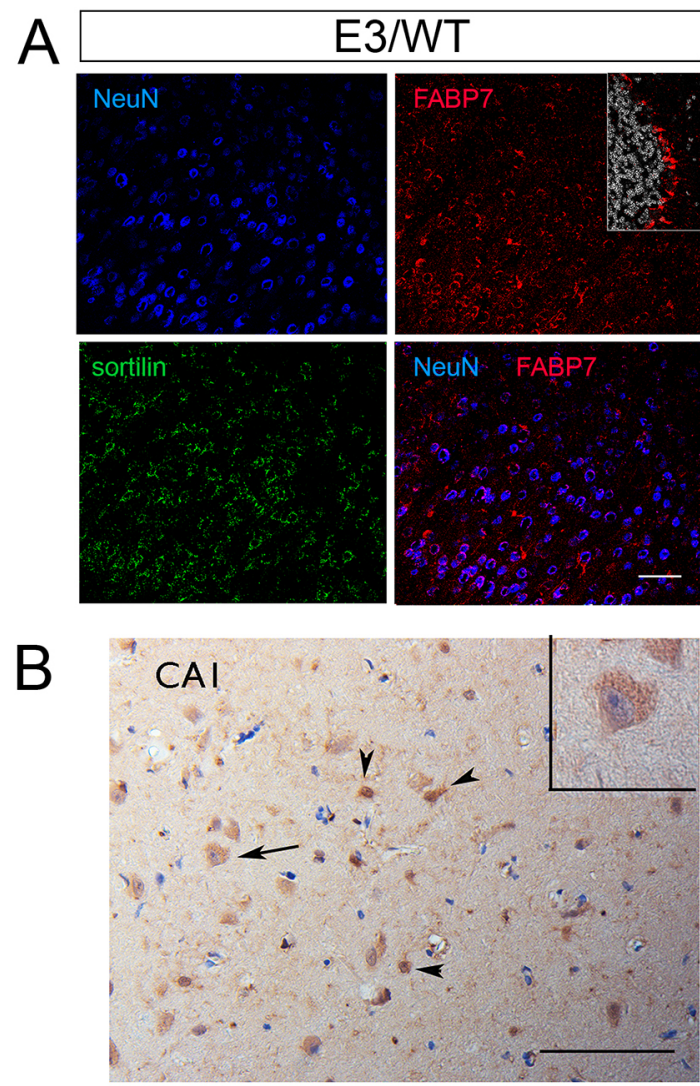
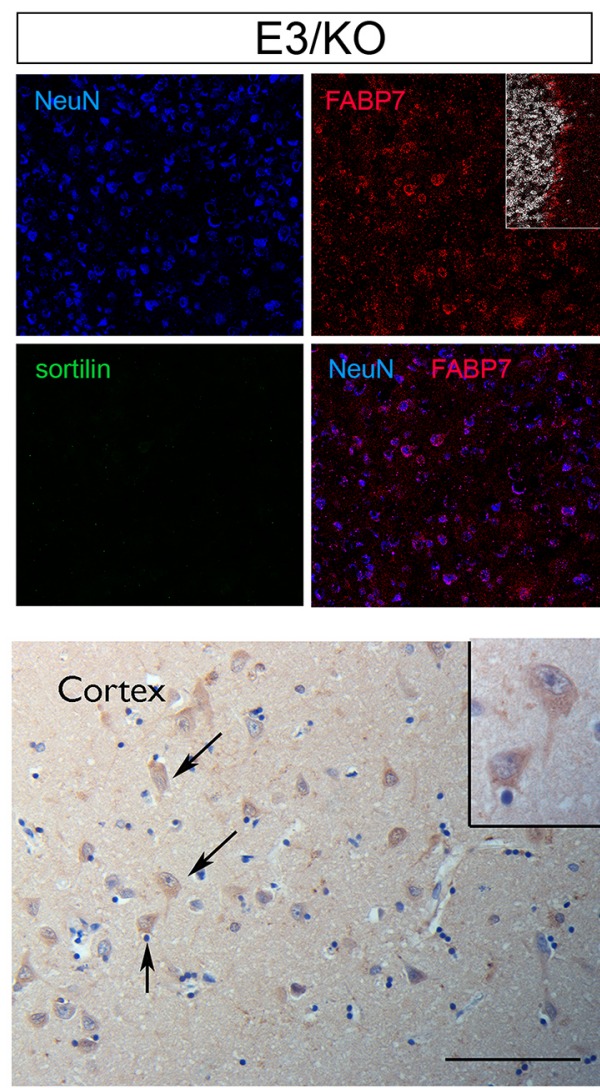

Fig. 5. Expression of FABP7 in neurons of mouse and human brains. (A) Immunohistological detection of FABP7 (red) in brain cortical sections from apoE3 mice, either wild-type (WT) or genetically deficient for Sort1 (KO). Additionally, the sections were stained for the neuronal marker NeuN (blue) and sortilin (green) as well as DAPI (white; in insets). Merged images show coexpression of FABP7 with NeuN. As a positive control, the insets document expression of FABP7 in glia in the cerebellum of E3/WT and E3/KO mice. Representative images from analysis of three mice per genotype are shown. Scale bar: $100 \mu \mathrm{m}$. (B) Staining for FABP7 in hippocampal subfield CA1 and temporal cortex specimens of $A D$ patients, showing immunoreactivity in glial cells (arrowheads) and light positivity in sparse neuronal cells (arrows, and insets). Representative images from one of three AD cases analyzed are shown (pathological characteristics given in Table S3). Scale bars: $100 \mu \mathrm{m}$.
Tripathi et al., 2017). Our data now document that FABP7 binds to sortilin and that this interaction controls stability and intracellular fate of the lipid carrier. Absence of sortilin alters the subcellular distribution of FABP7 and decreases its half-life, an effect seen in $\mathrm{CHO}$ cells, in primary neurons and in the brains of apoE3 mice deficient for sortilin. In recent transcriptomic analyses, no impact of apoE genotype on $\mathrm{Fabp} 7$ transcript levels was reported in the murine brain or human cerebral organoids (Zhao et al., 2020a,b). These findings agree with our data that sortilin impacts post-translational mechanisms of FABP7 expression control (Figs 4D and 6G). Given the importance of sortilin for functional expression of FABP7 in cells, and the detrimental impact of apoE4 on sortilin activity (Asaro et al., 2020), it is unclear why levels of the carrier in primary neurons of E4 mice increase in the absence of the receptor (compare E4/WT with E4/KO; Fig. 6D). Possibly, this phenomenon results from a secondary compensatory mechanism as suggested by the tendency for there to be increased $\mathrm{Fabp} 7$ transcript levels in E4/KO neurons as compared to all other genotype groups (Fig. 6G, $P=0.1028$ ).

In cells, FABP7 localizes to the cytoplasm, plasma membrane and nucleus, in line with its role in lipid sorting in these compartments. Apart from a nuclear localization signal (Liang et al., 2006), little is known about the mechanisms that direct this chaperone to distinct cellular organelles. Our data document that FABP7 directly interacts with sortilin, as shown by coimmunoprecipitation (Fig. 2A,B), and that it colocalizes with the receptor in endocytic and recycling compartments of the cell (Figs 2D and 7F-I). At present, we can only speculate about the molecular mechanism whereby sortilin affects trafficking of FABP7. However, an activity for sortilin in intracellular sorting of lipid transport particles has been shown in hepatocytes, where this receptor affects secretion of apoB-containing lipoproteins (Kjolby et al., 2010; Sparks et al., 2016; Strong et al., 2012).
FABP7 is perceived as a protein predominantly expressed in specialized glia such as Bergmann glia in the cerebellum or ependyma in brain cortex (Kaczocha et al., 2009; Kurtz et al., 1994; Owada, 2008). Our studies show co-expression of FABP7 with sortilin in neurons in culture (Fig. 6A) and in murine and human brains (Fig. 5). Undoubtedly, neuronal expression is modest as compared to glia expression. Still, given the low abundance of FABP7-expressing glia in cortex, one may conclude that neurons likely contribute the bulk of FABP7 mass detected in lysates of murine and human brain cortex (Fig. 4). Why sortilin deficiency impacts stability of FABP7 in neurons (Fig. 6) but not astrocytes (Fig. S1) is unclear at present, but lends further support for a molecular pathway unique to neuronal handling of PUFAs. Intriguingly, recent findings identified protein quality control by autophagy to be distinctly impacted in neurons but not astrocytes in AD (Bourdenx et al., 2021). Although not tested in this study, it is exciting to speculate that such a cell type-specific proteolytic mechanism may also define the stability of FABP7 in neurons versus astrocytes.

Loss of FABP7 activity in Fabp7 gene-targeted mice results in disturbances in brain lipid homeostasis including a decrease in DHA content (Owada et al., 2006). A comparable phenotype is seen in Sort1 KO mice that exhibit reduced levels of $\omega 3$-PUFA, DHA, as well as the eCB synaptamide (Asaro et al., 2020). As such changes are seen in the absence of any human APP transgenes in these mouse models, the effects of sortilin and apoE4 genotypes on brain lipid homeostasis are clearly not a secondary consequence of amyloidogenic processes. Whether $\mathrm{Fabp} 7$ mutant mice are prone to developing AD-like pathologies has not been investigated thus far. However, low levels of DHA and eCBs are correlated with $\mathrm{AD}$ pathologies in patients and mouse models (Jung et al., 2012; Koppel et al., 2009; Maroof et al., 2014; Schaefer et al., 2006; 

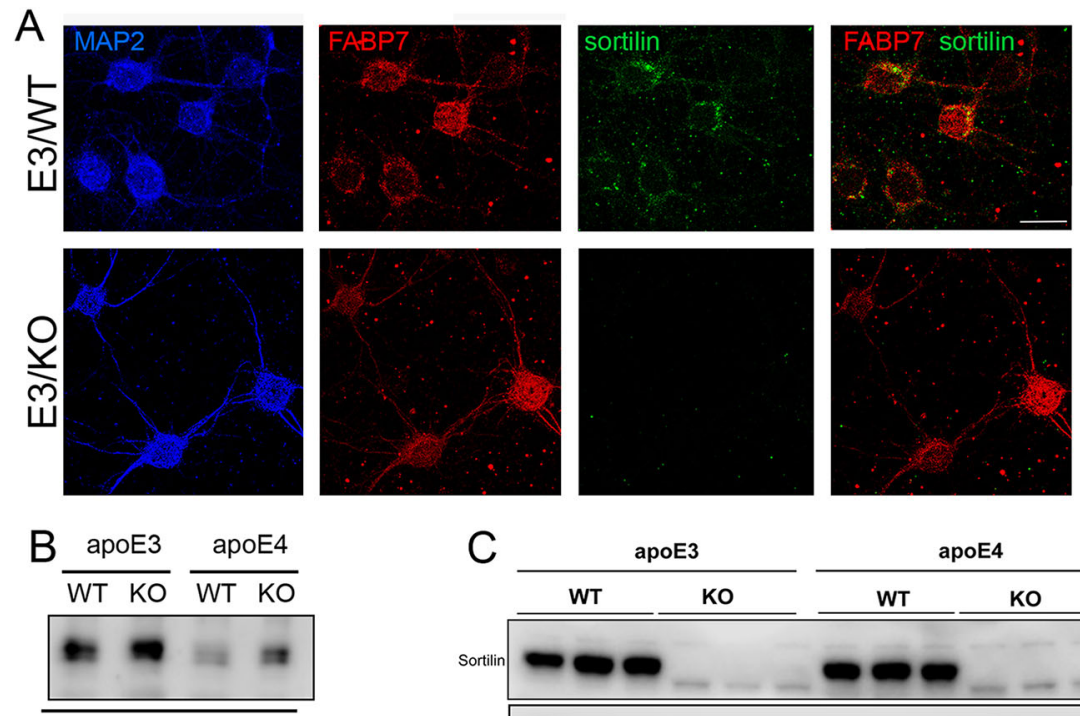

medium
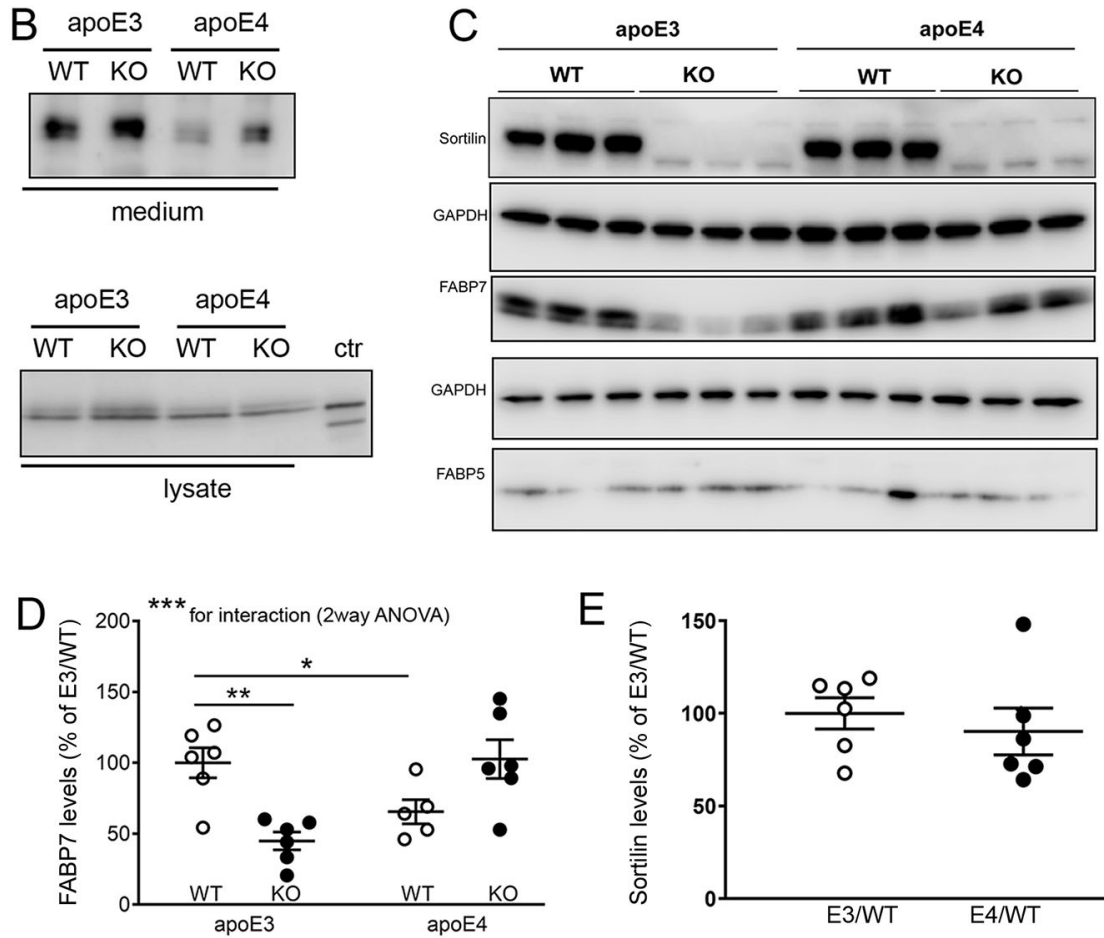

$E$

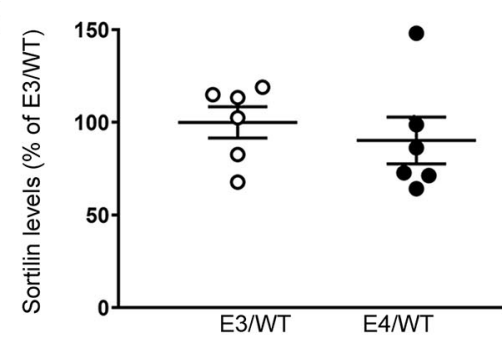

$\mathrm{F}$

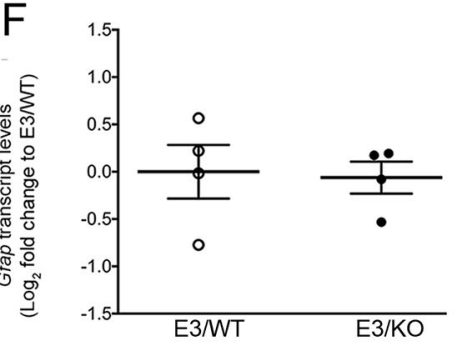

$\mathrm{G}$

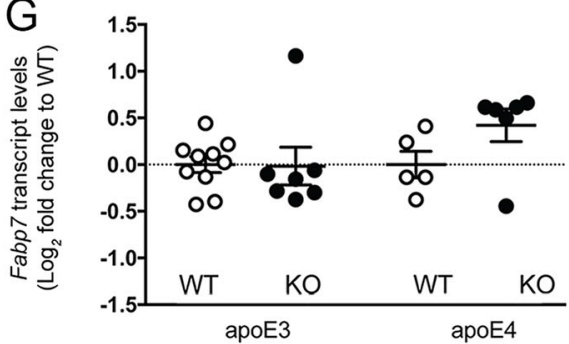

Fig. 6. Sortilin deficiency decreases FABP7 levels in primary neurons of apoE3 mice. (A) Immunodetection of FABP7 (red) and sortilin (green) in primary neuronal cultures from apoE3 mice either (WT) or genetically deficient for Sort1 (KO). FAPB7-stained cells are identified as neurons by expression of MAP2 (blue). Merged images show co-expression of FABP7 and sortilin. Images shown are representative of results from three independent experiments. Scale bar: $20 \mu \mathrm{m}$.

(B) Detection of apoE3 and apoE4 in medium and lysate of neuronal cultures of the indicated genotypes. Detection of recombinant apoE4 served as control (ctr). (C) Western blot analysis of sortilin as well as FABP5 and FABP7 in primary neuronal cultures from apoE3 or apoE4 expressing mice, either wild-type (WT) or genetically deficient for Sort1 (KO). Detection of GAPDH served as loading control. (D) Levels of FABP7 are reduced in primary neurons from E3/KO and E4/WT mice as compared with neurons from E3/WT animals as determined by densitometric scanning of replicate blots (exemplified in panel C). Data are given as mean \pm s.e.m. with the E3/WT levels set to $100 \%$ ( $n=8$ for E3 groups, $n=5$ for E4/WT, $n=4$ for E4/KO). ${ }^{* *} P<0.01$; ${ }^{* *} P<0.001$ for expression levels between E3/WT and all other genotypes (unpaired, two-tailed Student's $t$-test; ${ }^{*} P<0.05$ for the interaction of $A P O E$ and sortilin genotypes (two-way ANOVA with Bonferroni post test). (E) Levels of sortilin are comparable in primary neurons from E3/WT and E4/WT animals as determined by densitometric scanning of replicate blots (exemplified in panel C). Data are given as mean \pm s.e.m. with the E3/WT levels set to $100 \%(n=5-6$ biological replicates per genotype). (F) Quantitative RT-PCR analysis of Gfap transcripts in primary neuronal cultures from E3/WT and E3/KO mice. Values are given as $\log _{2}$ fold changes relative to E3/WT set to value 0 ( $n=4$ independent cultures per genotype). (G) Levels of Fabp7 transcript were determined in mouse primary neuronal cultures of the indicated genotypes by quantitative RT-PCR ( $n=10$ for E3/WT, $n=7$ for E3/KO, $n=5$ for E4/WT, $n=6$ for E4/ $\mathrm{KO})$. Values are given as $\log _{2}$ fold change relative to transcript levels in the respective WT (set to value 0).
Soderberg et al., 1991; Yuki et al., 2014), and so is low PPAR activity (Heneka et al., 2005; Pedersen et al., 2006). Jointly with the association of low FABP7 levels with the APOEe4 genotype in human $\mathrm{AD}$ specimens shown by us, these data argue for the significance of a lipid transport machinery involving sortilin, apoE3 and FABP7 in brain health, and defects in this machinery as a primary risk factor of sporadic AD.

\section{MATERIALS AND METHODS}

\section{Human specimens}

For expression analysis, brain autopsy specimens from the frontal cortex of AD patients were obtained from the Netherlands Brain Bank (Netherlands Institute for Neuroscience, Amsterdam) and the MRC London Brain Bank for Neurodegenerative Diseases (Institute of Psychiatry, King's College London). The ethnicity of samples was white. All material was collected from donors for or from whom a written informed consent for a brain autopsy and the use of the material and clinical information for research purposes had been obtained by the Netherlands Brain Bank or the MRC London Brain Bank. Detailed personal information, including age, gender, neuropathological stage, $A P O E$ genotype, of the individuals were provided by the brain banks (Table S2). Human post-mortem AD brain samples for immunohistochemistry were obtained from the archives of the Department of (Neuro) Pathology, Academic Medical Center (AMC), University of Amsterdam. Cases were selected from a retrospective searchable neuropathologic database, including cases with consent for post-mortem brain autopsy and use of their brain material and medical records for research (Table S3). The samples were used in compliance with 

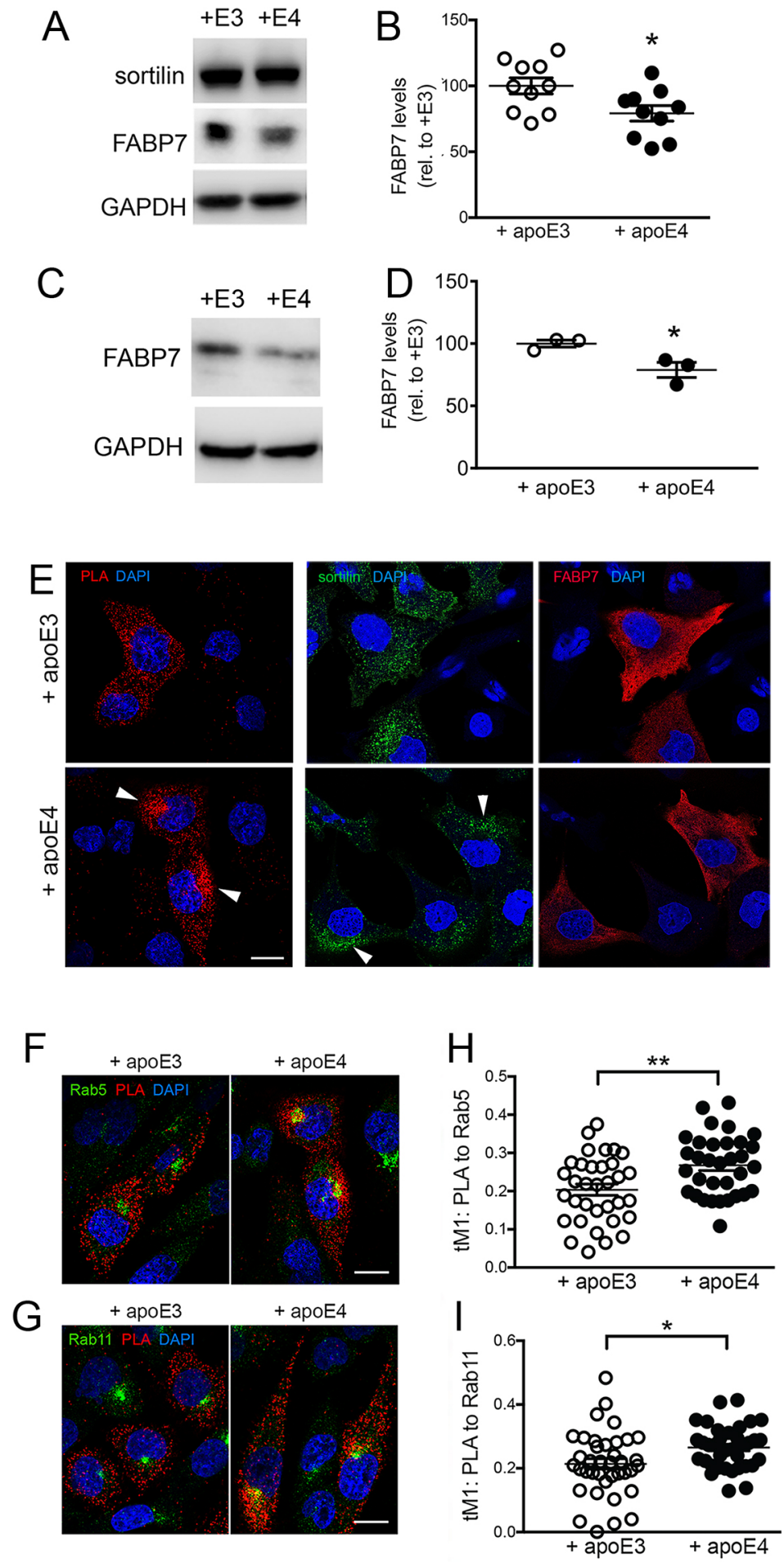

Fig. 7. Levels and intracellular sorting of FABP7 are impacted by application of apoE4. (A,B) $\mathrm{CHO}$ cells stably co-expressing sortilin and FABP7 (CHO-S/F) were treated for $24 \mathrm{~h}$ with conditioned medium containing $5 \mu \mathrm{g} / \mathrm{ml}$ of apoE3 (+E3) or apoE4 (+E4; see Materials and Methods for details). Western blot analysis (A) and densitometric scanning of replicate blots (B) document reduced levels of FABP7 in $\mathrm{CHO}-\mathrm{S} / \mathrm{F}$ cells in the presence of apoE4 as compared with apoE3 ( $n=10$ replicates for each condition from four independent experiments). Data are mean \pm s.e.m. given as percentage of FABP7 levels in apoE3-treated cells (set to $100 \%)$. ${ }^{*} P<0.05$ (unpaired, two-tailed Student's $t$-test). $(C, D)$ Experiment as in $A$ and $B$ but using conditioned medium from primary astrocytes secreting apoE3 or apoE4 ( $n=3$ replicates from one culture). Data are mean \pm s.e.m. given as percent of FABP7 levels in apoE3-treated cells (set to $100 \%$ ). ${ }^{*} P<0.05$ (unpaired, two-tailed Student's $t$-test). (E) Proximity ligation assay (PLA) to visualize the intracellular localization of sortilin-FABP7 complexes (red signal) in CHO-S/F cells treated with apoE3- or apoE4-conditioned HEK293 medium for $24 \mathrm{~h}$ (left panel). For comparison, immunostaining of total sortilin (middle panels) and FABP7 (right panels) in treated cells are shown as well. Cell nuclei were counterstained with DAPI (blue). PLA signals for sortilin-FABP7 complexes change from a dispersed vesicular pattern with apoE3 to a perinuclear pattern with apoE4 (arrowheads). A similar change is seen for total sortilin, whereas the pattern for total FABP7 remains unaffected by apoE4. Images shown are representative of results from three independent experiments. $(F, G)$ Colocalization studies in CHO-S/F cells documenting the presence of sortilin-FABP7 complexes (as deduced by PLA; red signal) in early endosomes, marked by antibodies against Rab5 (green signal; F) or recycling endosomes marked by Rab11 (green signal, G). (H,I) Extent of colocalization of PLA for sortilin and FABP7 with Rab5 $(H)(n=34$ cells for apoE3 and 33 cells for apoE4 treatment) and Rab11 (I) ( $n=38$ cells for each condition) as documented by thresholded Manders' coefficient tM1. This experiment was replicated three times. ${ }^{*} P<0.05 ;{ }^{* *}, P<0.01$ (unpaired, two-tailed Student's $t$-test). Scale bars: $20 \mu \mathrm{m}$. the Declaration of Helsinki 2000. The studies were approved by the Ethical Committees of the Academic Medical Center, Amsterdam (W11_073).

\section{Animal experimentation}

The generation of Sort1 ${ }^{-/-}$(Jansen et al., 2007) and Apoe targeted replacement (Knouff et al., 1999) strains of mice has been described. The animals were kept on normal chow ( $4.5 \%$ crude fat, $39 \%$ carbohydrates). All animal experimentation was conducted in male mice on an inbred C57B16/J background following approval by local ethics committees (X9017/17). The animals were 11 to 13 weeks of age.

\section{Surface proteome analysis}

Primary neurons from post natal mice (P0) were plated on poly-D-lysine coated plates $\left(5 \times 10^{6}\right.$ cells per $10 \mathrm{~cm}$ plate) and maintained in neurobasal medium (Invitrogen) supplemented with B27, glutamax, and penicillin/ streptomycin (Invitrogen) and used for experiments at days in vitro (DIV)10-12. Astrocytic cultures were prepared as previously published (Malik et al., 2020). Cells were placed in poly-L-lysine-coated flasks and cultured in Dulbecco's modified Eagle's medium (DMEM) with 10\% fetal bovine serum (FBS) and 1\% penicillin/streptomycin (Gibco, 15150122). After 10 DIV, microglia were removed by shaking and the astrocytes were plated for experiments. Biotinylation and purification of surface proteins 

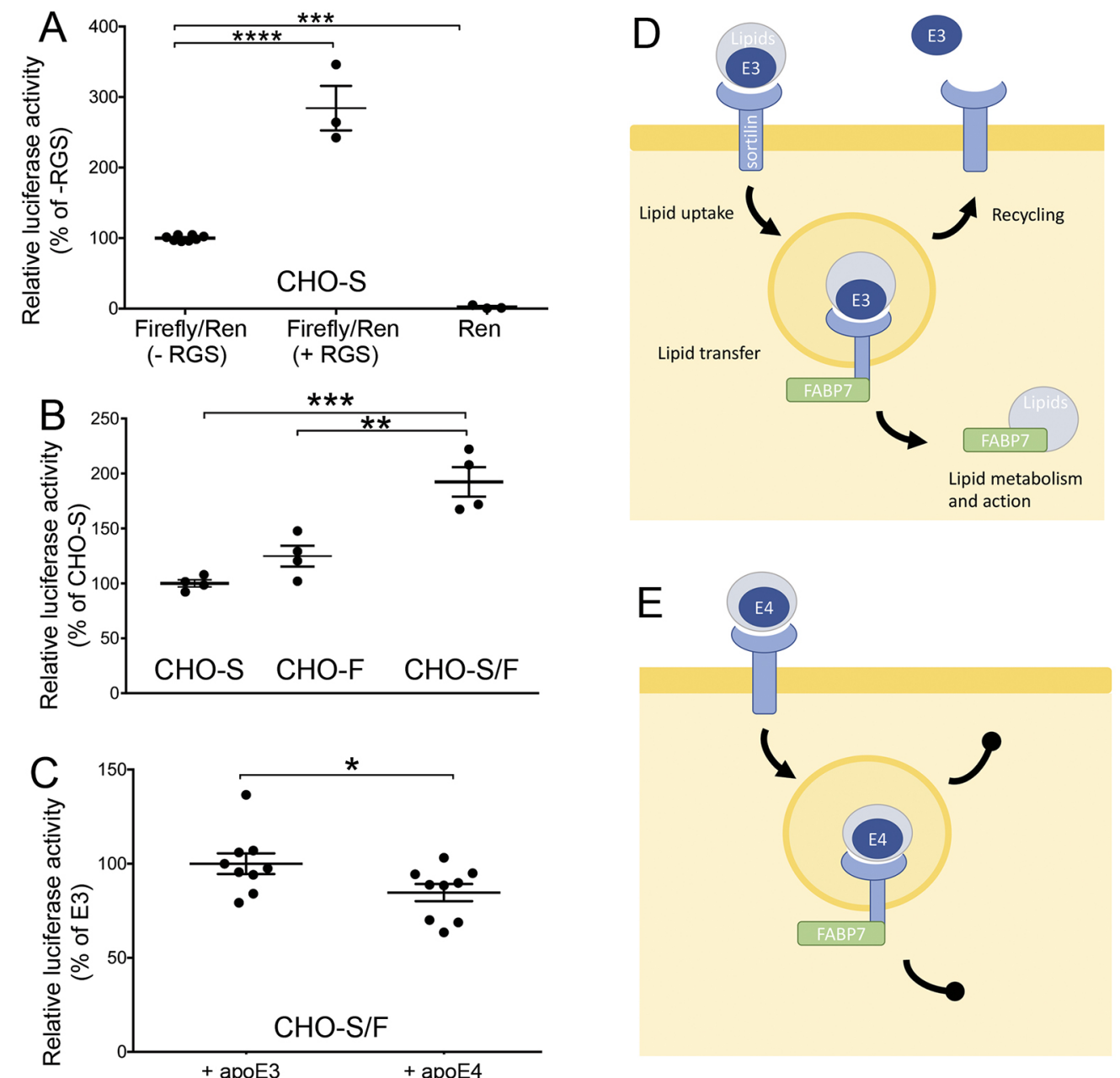

Fig. 8. Interaction of sortilin and FABP7 in PPAR $\gamma$-dependent gene expression. (A) CHO cells stably expressing sortilin (CHO-S) were transfected with reporter gene constructs encoding a PPAR-responsive firefly luciferase gene (Luc) and a constitutively expressed Renilla luciferase gene (Ren). Where indicated, transfectants were also treated with rosiglitazone (+RGS) for $24 \mathrm{~h}$. At $48 \mathrm{~h}$ after transfection, the activities of firefly and Renilla luciferases were determined in cell lysates using a luminometer ( $n=3-8$ replicates per cell line). Values are given as ratio of firefly to Renilla luciferase activity (mean \pm s.e.m., condition without RGS as $100 \%)$. ${ }^{* \star *} P<0.001 ;{ }^{* \star * *} P<0.0001$ (unpaired, two-tailed Student's $t$-test). (B) Replicate layers $(n=4)$ of $\mathrm{CHO}$ cells stably expressing sortilin (CHO-S), FABP7 (CHO-F), or both proteins (CHO-S/F) were transfected with constructs encoding a PPAR-responsive firefly luciferase and a constitutively expressed Renilla luciferase and analyzed for luciferase activity as described above. Values are given as ratio of firefly to Renilla luciferase (mean \pm s.e.m., CHO-S set to $100 \%$ ). ${ }^{* *} P<0.01 ;{ }^{* * *} P<0.001$ (unpaired, two-tailed Student's $t$-test). (C) CHO-S/F were transfected with reporter gene constructs encoding a PPAR-responsive firefly luciferase reporter gene and a constitutively expressed Renilla luciferase. Then, transfectants were treated with conditioned medium containing $5 \mu \mathrm{g} / \mathrm{ml}$ of human apoE3 or apoE4 for $24 \mathrm{~h}$. At $48 \mathrm{~h}$ after transfection, the activities of firefly and Renilla luciferases were determined in cell lysates using a luminometer ( $n=9$ replicates from 3 independent experiments per condition. Values are given as ratio of firefly to renilla luciferase (mean \pm s.e.m., + apoE 3 set to $100 \%)$. ${ }^{*} P<0.05$ (unpaired, two-tailed Student's $t$-test). (D,E) Proposed model of the interaction of sortilin with FABP7 and apoE3 (D) or apoE4 (E) in cellular lipid metabolism (see Discussion section for details).

was performed using EZ-Link ${ }^{\mathrm{TM}}$ Sulfo-NHS-SS-Biotin (Thermo Fisher Scientific) and Neutravidin slurry (Pierce) as described previously (Malik et al., 2019). For mass spectrometry analyses, proteins were reduced with $5 \mathrm{mM}$ Tris(2-chloroethyl)phosphine (TCEP) for $60 \mathrm{~min}$ at $60^{\circ} \mathrm{C}$. To block reduced cysteines, $200 \mathrm{mM}$ methyl methanethiosulfonate (MMTS) at a final concentration of $10 \mathrm{mM}$ was added and the sample was incubated at room temperature for $10 \mathrm{~min}$. Proteins were digested overnight with $10 \mathrm{ng} / \mu \mathrm{l}$ trypsin and the resulting peptides were analyzed by LC-MS/MS for peptide identification and LC-MS for relative quantification using a Q Exactive mass spectrometer (Thermo Fisher Scientific) coupled with a nanoAcquity LC system (Waters). To do so, the samples were desalted and concentrated on a nanoACQUITY UPLC Trapping Column (Waters). Further peptide separation was carried on a nanoACQUITY UPLC BEH C18 Column (Waters, $75 \mu \mathrm{m}$ inner diameter; $250 \mathrm{~mm}$ long) with an acetonitrile gradient (5-35\% over $160 \mathrm{~min}$ ) in the presence of $0.1 \%$ formic acid at a flow rate of $250 \mathrm{nl} / \mathrm{min}$. Qualitative LC-MS/MS measurements (i.e. peptide and protein identification) were carried out on samples pooled within each experimental group. Quantitative analyses of individual samples used separate survey scan LC-MS runs.

Pre-processed LC-MS/MS data files were submitted to the Mascot search engine (Matrix Science) and searched against Mus musculus protein entries from the SwissProt database using a target/decoy approach. The search parameters were as follows: enzyme semitrypsin; number of missed cleavages: 1; ion mass error tolerances: $\pm 5 \mathrm{ppm}$ (parent) and $\pm 0.01 \mathrm{Da}$ (fragment); modifications: methylthio $\mathrm{C}$ (fixed), oxidation $\mathrm{M}$ (variable). The statistical significance of the identifications was assessed as described previously (Kall et al., 2008). Only peptide sequences with $q$-values $\leq 0.01$ were considered as confidently identified.

Peptides identified in the LC-MS/MS analysis were quantified in individual LC-MS samples using a feature extraction procedure (Bakun et al., 2009). The calculated peptide abundances were log-transformed, normalized by a robust locally weighted regression smoother (LOESS) 
(Cleveland and Devlin, 1988), and rolled-up to relative protein abundances. To select differentially expressed proteins, a resampling ANOVA-based significance test was used.

\section{Immunohistochemistry}

For staining of human brain tissue, 3-4- $\mu \mathrm{m}$ paraffin sections were placed on poly-L-lysine coated slides and allowed to dry in an oven $\left(37^{\circ} \mathrm{C}\right)$ overnight and then processed for immunohistochemistry described in detail elsewhere (Jesse et al., 2017). The sections were deparaffinized in xylene for $20 \mathrm{~min}$ and rehydrated in $100 \%, 96 \%$ and $75 \%$ ethanol for 3 min each followed by endogenous peroxidase quenching $\left(0.3 \% \mathrm{H}_{2} \mathrm{O}_{2}\right.$ in methanol) for $20 \mathrm{~min}$. For antigen retrieval, sections were heated in citrate buffer, $\mathrm{pH} 6$ (DAKO), for $10 \mathrm{~min}$ in a pressure cooker. After washing in PBS, sections were incubated with primary antibody directed against FABP7 (kindly provided by Thomas Müller, MDC Berlin, Germany) at $4^{\circ} \mathrm{C}$ overnight. After washing in PBS, sections were incubated with secondary antibody (IL Immunologic, Duiven, The Netherlands) for $30 \mathrm{~min}$ at room temperature. DAB reagent (BrightDAB substrate kit; ImmunoLogic, Duiven, The Netherlands) was used to visualize antibody binding. The sections were then counter-stained with $6 \%$ hematoxylin for $3 \mathrm{~min}$. All procedures were performed at room temperature.

For immunohistology of the murine brain, $10 \mu \mathrm{m}$ coronal cryosections were incubated in blocking buffer $(0.5 \%$ BSA, $10 \%$ donkey serum and $0.3 \%$ triton) for $1 \mathrm{~h}$. The sections were subsequently washed in PBS-T $(1 \times \mathrm{PBS}$, $0.1 \%$ Triton $\mathrm{X}-100)$ for $10 \mathrm{~min}$, followed by immunostaining with primary antibodies (diluted in blocking buffer) directed against NeuN (Abcam, \#ab104224; 1:500), FABP7 (EMD Millipore, \#ABN14; 1:200) and sortilin (R\&D Systems, \#AF2934; 1:200), followed by incubation with fluorophoreconjugated secondary antibodies (conjugated to Alexa Fluor 488, 555, and 647 diluted 1:1000). The sections were washed, stained with DAPI (1:3000), and mounted onto glass slides for standard confocal microscopy.

\section{Expression analysis}

Determinations of transcript and protein levels were performed by quantitative RT-PCR and SDS-PAGE, respectively, using standard protocols. Probes for transcript analyses were Taqman Gapdh (Mm99999915_g1), Fabp 7 (Mm00445225_m1), and Gfap (Mm01253033_m1). Fold change in transcript levels was calculated using the cycle threshold (CT) comparative method $\left(2^{-\mathrm{ddCT}}\right)$ normalizing to CT values of internal control Gapdh gene. For semi-quantitative western blot analysis, the intensities of immunoreactive signals were quantified by densitometric scanning of blots using the Image Studio Lite software. Signal intensities were always normalized to loading controls detected on the same membrane.

Primary antibodies used for immunodetection were directed against $\beta$-tubulin (abcam, ab6046; Millipore, CP06; 1:1000), FABP7 (Millipore, ABN14), FABP5 (Biozol Diagnostica, BVD-RD181060100-1; 1:1000), GAPDH (Genetex, GTX627408-01; 1:1000), apoE (Millipore, AB947; 1:1000), and sortilin (BD Transduction Laboratories, 612101; 1:1000).

\section{Cell culture experiments}

Chinese hamster ovary cells stably overexpressing FABP7 and/or murine sortilin were generated in-house from the parental line CHO-K1 (ATTC \# CCL-61). Parental Chinese hamster ovary (CHO-K1) cells or cell clones stably overexpressing mouse sortilin and/or Myc-tagged mouse FABP7 (Origene, MR200772) were grown in DMEM (Gibco) supplemented with $10 \%$ FCS (Gibco) and 1\% penicillin/streptomycin (Gibco). HEK293 EBNA cells were purchased from ATTC (\#CRL-1573). All cell lines were routinely checked for absence of mycoplasma. To generate medium containing human apoE3 or apoE4, HEK293 cells were transiently transfected with expression constructs encoding for human apoE3 or apoE4. Cell supernatants were conditioned for $48 \mathrm{~h}$ in medium without fetal calf serum (FCS) (Chen et al., 2010). Alternatively, supernatants from primary astrocytes were conditioned for $48 \mathrm{~h}$ in medium without FCS. The concentration of apoE in media batches from HEK293 or primary astrocytes was determined by western blot analysis comparing apoE signal intensities to those from serial dilutions of recombinant human apoE (MBL, JM-4699-500) included in the same blots.
For western blot and qRT-PCR analyses, neuronal or glial cultures were used at DIV 10-12. For immunocytochemistry, cells plated on coverslips were fixed with $4 \%$ paraformaldehyde (PFA), incubated overnight at $4{ }^{\circ} \mathrm{C}$ with primary antibodies directed against MAP2 (Synaptic systems, \#188004; 1:250), FABP7 (EMD Millipore, \#ABN14; 1:100) and sortilin (R\&D Systems, \#AF2934; 1:100), followed by incubation with fluorophore-labeled secondary antibodies (conjugated to Alexa Fluor 488, 555 and 647; diluted 1:1000) and mounting onto glass slides using fluorescent mounting medium (Dako). Cell lysates for western blot analysis were generated using standard protocols

\section{PPAR-dependent luciferase assay}

To investigate the transcriptional activity of PPARs, we used a peroxisome proliferator response element (PPRE)-driven luciferase reporter gene approach (Kim et al., 1998). In brief, CHO cell lines were transfected with a reporter construct containing three copies of the PPRE placed upstream of the thymidine kinase promoter- firefly luciferase fusion gene (PPRE X3-TK-luc, Addgene plasmid \#1015). In addition, the cells were transfected with a cytomegalovirus promoter-driven Renilla luciferase reporter gene (pGL4.75 hRluc/CMV, Promega, E6931) used as transfection control. Where indicated, transfectants were treated with $5 \mu \mathrm{M}$ of rosiglitazone (Sigma, R2408) or with conditioned medium containing $5 \mu \mathrm{g} / \mathrm{ml}$ of human apoE3 or apoE4 for $24 \mathrm{~h}$. At 24 to $48 \mathrm{~h}$ after transfection, cells were lysed in buffer $(50 \mathrm{mM}$ Tris-HCl, $\mathrm{pH} 7.4,140 \mathrm{mM} \mathrm{NaCl}$ and $1 \%$ Triton X-100) and lysates used to assay luciferase activity in 96-well plates using the Dual-Luciferase Reporter Assay (Promega) according to the manufacturer's instructions. The luminescence-based PPAR activity (firefly luciferase) was normalized to the internal Renilla luciferase activity.

\section{Protein interaction studies}

Proximity ligation is an experimental approach to test the spatial proximity of proteins in cells. It employs oligonucleotide-conjugated antibodies directed against the target proteins (here FABP7-Myc and sortilin). Here, CHO-S/F cells stably overexpressing mouse sortilin and Myc-tagged mouse FABP7 were grown on glass coverslips and treated for $24 \mathrm{~h}$ with conditioned medium containing $5 \mu \mathrm{g} / \mathrm{ml}$ of human apoE3 or apoE4. Then, the cells were fixed with $4 \%$ PFA in PBS and subjected to proximity ligation assay (PLA) using orange PLA probes, followed by ligation and amplification performed according to the manufacturer's recommendation (Sigma). Thereafter, the cells were incubated with primary and secondary antibodies to co-stain for the indicated marker proteins (anti-Rab5, Cell Signaling, \#2143, 1:500; anti-Rab11, Cell Signaling, \#5589, 1:500; and Alexa Fluor 647-conjugated secondary antibodies). Microscopy images were quantified blinded using Fiji software. Colocalization analysis was performed with the Colocalization Threshold plugin with manual selection of the region of interest. Single $z$-plane images were used for calculating the thresholded Manders' coefficient (tM1). Analysis of protein localization by subcellular fractionation was carried out as previously described (Andersen et al., 2005).

\section{Statistical analysis}

For all in vivo experiments, an indicated number $n$ is the number of mice per group used in an experiment. For primary culture experiments, an indicated number $n$ is the number of independent neuronal or glial preparations (biological replicates) used for western blotting or qRT-PCR analyses. For colocalization studies in CHO cells, $n$ is the number of cells analyzed in replicate experiments. Each mouse or human specimen, or biological replicate in a cell culture experiment represents a statistically independent experimental unit, which was treated accordingly as an independent value in the statistical analyses. Statistical analyses were performed using GraphPad Prism software. For data with two different conditions and/or time points, two-way ANOVA with Bonferroni multiple comparisons test was applied. To select differentially expressed proteins in the surface proteome analysis, a resampling ANOVA-based significance test was used. Further details of statistical analyses not included here are specified in the respective figure legends. 


\section{Acknowledgement}

We are indebted to T. Pasternack, K. Kampf, K. M. Pedersen, and C. Kruse for expert technical assistance. We also wish to thank H. Riezman (University of Geneva) for support and J. Bosset (Bioimaging Center UNIGE) for help with microscopy.

\section{Competing interests}

The authors declare no competing or financial interests.

\section{Author contributions}

Conceptualization: A.A., R.S., M.B., O.K., A.-S.C.-S., T.R., A.R., M.D., B.K., E.A A.R.M., T.W.; Methodology: A.A., R.S., M.B., O.K., A.-S.C.-S., A.R., A.R.M.; Validation: A.A., R.S., M.B., O.K., A.-S.C.-S., T.R., A.R., M.D., B.K., E.A., A.R.M., T.W.; Investigation: A.A., R.S., M.B., O.K., A.-S.C.-S., A.R., A.R.M.; Resources: T.R., M.D., T.W.; Data curation: A.A., A.R.M.; Writing - original draft: T.W.; Writing review \& editing: T.W.; Supervision: M.D., B.K., E.A., A.R.M., T.W.; Project administration: T.R., M.D., B.K., E.A., T.W.; Funding acquisition: T.R., M.D., B.K. E.A., T.W.

\section{Funding}

Studies were funded in part by the European Research Council (ERC) (BeyOND No. 335692), the Helmholtz Association (AMPro), the Alzheimer Forschung Initiative (\#18003), and the Novo Nordisk Foundation (NNF18OC0033928) to T.E.W.; and by the Foundation for Polish Science (Fundacja na rzecz Nauki Polskiej) co-financed by the European Union under the European Regional Development Fund (Homing program, POIR.04.04.00 00 5CEF/18 00) to A.R.M. Equipment for surface proteomics was provided in part by the Centre for Preclinical Research and Technology (CePT), co-sponsored by the European Regional Development Fund and Innovative Economy, The National Cohesion Strategy of Poland. Deposited in PMC for immediate release.

\section{Peer review history}

The peer review history is available online at https://journals.biologists.com/jcs/ article-lookup/doi/10.1242/jcs.258894

\section{References}

Adida, A. and Spener, F. (2006). Adipocyte-type fatty acid-binding protein as intercompartmental shuttle for peroxisome proliferator activated receptor $\gamma$ agonists in cultured cell. Biochim. Biophys. Acta (BBA) Mol. Cell Biol. Lipids 1761, 172-181. doi:10.1016/j.bbalip.2006.02.006

Al-Akhrass, H., Naves, T., Vincent, F., Magnaudeix, A., Durand, K., Bertin, F. Melloni, B., Jauberteau, M.-O. and Lalloué, F. (2017). Sortilin limits EGFR signaling by promoting its internalization in lung cancer. Nat. Commun. 8, 1182 doi:10.1038/s41467-017-01172-5

Andersen, O. M., Reiche, J., Schmidt, V., Gotthardt, M., Spoelgen, R., Behlke, J., von Arnim, C. A. F., Breiderhoff, T., Jansen, P., Wu, X. et al. (2005). Neuronal sorting protein-related receptor sorLA/LR11 regulates processing of the amyloid precursor protein. Proc. Natl. Acad. Sci. USA 102, 13461-13466. doi:10.1073/pnas.0503689102

Asaro, A., Carlo-Spiewok, A. S., Malik, A. R., Rothe, M., Schipke, C. G., Peters, O., Heeren, J. and Willnow, T. E. (2020). Apolipoprotein E4 disrupts the neuroprotective action of sortilin in neuronal lipid metabolism and endocannabinoid signaling. Alzheimer's Dement. 16, 1248-1258. doi:10.1002/ alz.12121

Bakun, M., Karczmarski, J., Poznanski, J., Rubel, T., Rozga, M., Malinowska, A., Sands, D., Hennig, E., Oledzki, J., Ostrowski, J. et al. (2009). An integrated LC ESI-MS platform for quantitation of serum peptide ladders. Application for colon carcinoma study. Proteomics Clin. Appl. 3, 932-946. doi:10.1002/prca.200800111

Balendiran, G. K., Schnütgen, F., Scapin, G., Börchers, T., Xhong, N., Lim, K., Godbout, R., Spener, F. and Sacchettini, J. C. (2000). Crystal structure and thermodynamic analysis of human brain fatty acid-binding protein. J. Biol. Chem 275, 27045-27054. doi:10.1016/S0021-9258(19)61478-X

Bourdenx, M., Martin-Segura, A., Scrivo, A., Rodriguez-Navarro, J. A., Kaushik, S., Tasset, I., Diaz, A., Storm, N. J., Xin, Q., Juste, Y. R. et al. (2021). Chaperone-mediated autophagy prevents collapse of the neuronal metastable proteome. Cell 184, 2696-2714.e25. doi:10.1016/j.cell.2021.03.048

Butkinaree, C., Canuel, M., Essalmani, R., Poirier, S., Benjannet, S., Asselin, M.-C., Roubtsova, A., Hamelin, J., Marcinkiewicz, J., Chamberland, A. et al. (2015). Amyloid precursor-like protein 2 and sortilin do not regulate the PCSK9 convertase-mediated low density lipoprotein receptor degradation but interact with each other. J. Biol. Chem. 290, 18609-18620. doi:10.1074/jbc.M115.647180

Carlo, A.-S., Gustafsen, C., Mastrobuoni, G., Nielsen, M. S., Burgert, T., Hartl, D., Rohe, M., Nykjaer, A., Herz, J., Heeren, J. et al. (2013). The proneurotrophin receptor sortilin is a major neuronal apolipoprotein $\mathrm{E}$ receptor for catabolism of amyloid- $\beta$ peptide in the brain. J. Neurosci. 33, 358-370 doi:10.1523/JNEUROSCI.2425-12.2013
Carlo, A.-S., Nykjaer, A. and Willnow, T. E. (2014). Sorting receptor sortilin-a culprit in cardiovascular and neurological diseases. J. Mol. Med. 92, 905-911. doi:10.1007/s00109-014-1152-3

Chen, Y., Durakoglugil, M. S., Xian, X. and Herz, J. (2010). ApoE4 reduces glutamate receptor function and synaptic plasticity by selectively impairing ApoE receptor recycling. Proc. Natl. Acad. Sci. USA 107, 12011-12016. doi:10.1073/ pnas.0914984107

Cleveland, W. S. and Devlin, S. J. (1988). Locally weighted regression: an approach to regression analysis by local fitting. J. Am. Stat. Assoc. 83, 596-610. doi:10.1080/01621459.1988.10478639

Corder, E. H., Saunders, A. M., Strittmatter, W. J., Schmechel, D. E., Gaskell, P. C., Small, G. W., Roses, A. D., Haines, J. L. and PericakVance, M. A. (1993). Gene dose of apolipoprotein E type 4 allele and the risk of Alzheimer's disease in late onset families. Science 261, 921-923. doi:10.1126/ science. 8346443

Elmes, M. W., Kaczocha, M., Berger, W. T., Leung, K. N., Ralph, B. P., Wang, L Sweeney, J. M., Miyauchi, J. T., Tsirka, S. E., Ojima, I. et al. (2015). Fatty acidbinding proteins (FABPs) are intracellular carriers for $\Delta^{9}$-tetrahydrocannabino (THC) and cannabidiol (CBD). J. Biol. Chem. 290, 8711-8721. doi:10.1074/jbc. M114.618447

Feng, L., Hatten, M. E. and Heintz, N. (1994). Brain lipid-binding protein (BLBP): a novel signaling system in the developing mammalian CNS. Neuron 12, 895-908. doi:10.1016/0896-6273(94)90341-7

Heneka, M. T., Sastre, M., Dumitrescu-Ozimek, L., Hanke, A., Dewachter, I, Kuiperi, C., O'Banion, K., Klockgether, T., Van Leuven, F. and Landreth, G. E. (2005). Acute treatment with the PPAR $\gamma$ agonist pioglitazone and ibuprofen reduces glial inflammation and A $\beta 1-42$ levels in APPV717I transgenic mice. Brain 128, 1442-1453. doi:10.1093/brain/awh452

Holtzman, D. M., Herz, J. and Bu, G. (2012). Apolipoprotein e and apolipoprotein receptors: normal biology and roles in Alzheimer disease. Cold Spring Harb. Perspect. Med. 2, a006312. doi:10.1101/cshperspect.a006312

Hu, F., Padukkavidana, T., Vægter, C. B., Brady, O. A., Zheng, Y. Mackenzie, I. R., Feldman, H. H., Nykjaer, A. and Strittmatter, S. M. (2010) Sortilin-mediated endocytosis determines levels of the frontotemporal dementia protein, progranulin. Neuron 68,654-667. doi:10.1016/j.neuron.2010.09.034

Jansen, P., Giehl, K., Nyengaard, J. R., Teng, K., Lioubinski, O. Sjoegaard, S. S., Breiderhoff, T., Gotthardt, M., Lin, F., Eilers, A. et al. (2007). Roles for the pro-neurotrophin receptor sortilin in neuronal development, aging and brain injury. Nat. Neurosci. 10, 1449-1457. doi:10.1038/nn2000

Jesse, C. M., Bushuven, E., Tripathi, P., Chandrasekar, A., Simon, C. M., Drepper, C., Yamoah, A., Dreser, A., Katona, I., Johann, S. et al. (2017). ALS associated endoplasmic reticulum proteins in denervated skeletal muscle: implications for motor neuron disease pathology. Brain Pathol. 27, 781-794. doi:10.1111/bpa.12453

Jung, K.-M., Astarita, G., Yasar, S., Vasilevko, V., Cribbs, D. H., Head, E. Cotman, C. W. and Piomelli, D. (2012). An amyloid $\beta_{42}$-dependent deficit in anandamide mobilization is associated with cognitive dysfunction in Alzheimer's disease. Neurobiol. Aging 33, 1522-1532. doi:10.1016/j.neurobiolaging.2011.03. 012

Kaczocha, M., Glaser, S. T. and Deutsch, D. G. (2009). Identification of intracellular carriers for the endocannabinoid anandamide. Proc. Natl. Acad. Sci. USA 106, 6375-6380. doi:10.1073/pnas.0901515106

Käll, L., Storey, J. D., MacCoss, M. J. and Noble, W. S. (2008). Assigning significance to peptides identified by tandem mass spectrometry using decoy databases. J. Proteome Res. 7, 29-34. doi:10.1021/pr700600n

Kim, J. B., Wright, H. M., Wright, M. and Spiegelman, B. M. (1998). ADD1/ SREBP1 activates PPARgamma through the production of endogenous ligand Proc. Natl. Acad. Sci. USA 95, 4333-4337. doi:10.1073/pnas.95.8.4333

Kim, E., Lee, Y., Lee, H.-J., Kim, J. S., Song, B.-S., Huh, J.-W., Lee, S.-R., Kim, S.U., Kim, S.-H., Hong, Y. et al. (2010). Implication of mouse Vps26b-Vps29-Vps35 retromer complex in sortilin trafficking. Biochem. Biophys. Res. Commun. 403 167-171. doi:10.1016/j.bbrc.2010.10.121

Kjolby, M., Andersen, O. M., Breiderhoff, T., Fjorback, A. W., Pedersen, K. M. Madsen, P., Jansen, P., Heeren, J., Willnow, T. E. and Nykjaer, A. (2010). Sort1, encoded by the cardiovascular risk locus $1 \mathrm{p} 13.3$, is a regulator of hepatic lipoprotein export. Cell Metab. 12, 213-223. doi:10.1016/j.cmet.2010.08.006

Knouff, C., Hinsdale, M. E., Mezdour, H., Altenburg, M. K., Watanabe, M., Quarfordt, S. H., Sullivan, P. M. and Maeda, N. (1999). Apo E structure determines VLDL clearance and atherosclerosis risk in mice. J. Clin Invest 103 1579-1586. doi:10.1172/JCl6172

Koppel, J., Bradshaw, H., Goldberg, T. E., Khalili, H., Marambaud, P., Walker, M. J., Pazos, M., Gordon, M. L., Christen, E. and Davies, P. (2009) Endocannabinoids in Alzheimer's disease and their impact on normative cognitive performance: a case-control and cohort study. Lipids Health Dis. 8, 2 . doi:10.1186/1476-511X-8-2

Kurtz, A., Zimmer, A., Schnutgen, F., Bruning, G., Spener, F. and Muller, T. (1994). The expression pattern of a novel gene encoding brain-fatty acid binding protein correlates with neuronal and glial cell development. Development 120 2637-2649. doi:10.1242/dev.120.9.2637 
Liang, Y., Bollen, A. W., Aldape, K. D. and Gupta, N. (2006). Nuclear FABP7 immunoreactivity is preferentially expressed in infiltrative glioma and is associated with poor prognosis in EGFR-overexpressing glioblastoma. BMC Cancer 6, 97 doi:10.1186/1471-2407-6-97

Lv, Y., Yang, J., Gao, A., Sun, S., Zheng, X., Chen, X., Wan, W., Tang, C., Xie, W. Li, S. et al. (2019). Sortilin promotes macrophage cholesterol accumulation and aortic atherosclerosis through lysosomal degradation of ATP-binding cassette transporter A1 protein. Acta Biochim. Biophys. Sin. 51, 471-483. doi:10.1093/ abbs/gmz029

Malik, A. R., Szydlowska, K., Nizinska, K., Asaro, A., van Vliet, E. A., Popp, O. Dittmar, G., Fritsche-Guenther, R., Kirwan, J. A., Nykjaer, A. et al. (2019). SorCS2 controls functional expression of amino acid transporter EAAT3 and protects neurons from oxidative stress and epilepsy-induced pathology. Cell Rep. 26, 2792-2804.e6. doi:10.1016/j.celrep.2019.02.027

Malik, A. R., Lips, J., Gorniak-Walas, M., Broekaart, D. W. M., Asaro, A., Kuffner, M. T. C., Hoffmann, C. J., Kikhia, M., Dopatka, M., Boehm-Sturm, P. et al. (2020). SorCS2 facilitates release of endostatin from astrocytes and controls post-stroke angiogenesis. Glia 68, 1304-1316. doi:10.1002/glia.23778

Maroof, N., Ravipati, S., Pardon, M. C., Barrett, D. A. and Kendall, D. A. (2014) Reductions in endocannabinoid levels and enhanced coupling of cannabinoid receptors in the striatum are accompanied by cognitive impairments in the A $\beta P P s w e / P S 1 \triangle E 9$ mouse model of Alzheimer's disease. J. Alzheimer's Dis. 42 227-245. doi:10.3233/JAD-131961

Matsumata, M., Inada, H. and Osumi, N. (2016). Fatty acid binding proteins and the nervous system: their impact on mental conditions. Neurosci. Res. 102, 47-55 doi:10.1016/j.neures.2014.08.012

Mita, R., Beaulieu, M. J., Field, C. and Godbout, R. (2010). Brain fatty acid-binding protein and $\omega-3 / \omega-6$ fatty acids: mechanistic insight into malignant glioma cell migration. J. Biol. Chem. 285, 37005-37015. doi:10.1074/jbc.M110.170076

Moullé, V. S. F., Cansell, C., Luquet, S. and Cruciani-Guglielmacci, C. (2012). The multiple roles of fatty acid handling proteins in brain. Front. Physiol. 3, 385 doi:10.3389/fphys.2012.00385

Ni, X. and Morales, C. R. (2006). The lysosomal trafficking of acid sphingomyelinase is mediated by sortilin and mannose 6-phosphate receptor. Traffic 7, 889-902. doi:10.1111/j.1600-0854.2006.00429.x

Nielsen, M. S., Madsen, P., Christensen, E. I., Nykjær, A., Gliemann, J., Kasper, D., Pohlmann, R. and Petersen, C. M. (2001). The sortilin cytoplasmic tail conveys Golgi-endosome transport and binds the VHS domain of the GGA2 sorting protein. EMBO J. 20, 2180-2190. doi:10.1093/emboj/20.9.2180

Owada, Y. (2008). Fatty acid binding protein: localization and functional significance in the brain. Tohoku J. Exp. Med. 214, 213-220. doi:10.1620/tjem.214.213

Owada, Y., Abdelwahab, S. A., Kitanaka, N., Sakagami, H., Takano, H., Sugitani, Y., Sugawara, M., Kawashima, H., Kiso, Y., Mobarakeh, J. I. et al. (2006). Altered emotional behavioral responses in mice lacking brain-type fatty acid-binding protein gene. Eur. J. Neurosci. 24, 175-187. doi:10.1111/j.1460 9568.2006.04855.x

Pedersen, W. A., McMillan, P. J., Kulstad, J. J., Leverenz, J. B., Craft, S. and Haynatzki, G. R. (2006). Rosiglitazone attenuates learning and memory deficits in
Tg2576 Alzheimer mice. Exp. Neurol. 199, 265-273. doi:10.1016/j.expneurol. 2006.01.018

Schaefer, E. J., Bongard, V., Beiser, A. S., Lamon-Fava, S., Robins, S. J., Au, R. Tucker, K. L., Kyle, D. J., Wilson, P. W. F. and Wolf, P. A. (2006). Plasma phosphatidylcholine docosahexaenoic acid content and risk of dementia and Alzheimer disease: the framingham heart study. Arch. Neurol. 63, 1545-1550. doi:10.1001/archneur.63.11.1545

Söderberg, M., Edlund, C., Kristensson, K. and Dallner, G. (1991). Fatty acid composition of brain phospholipids in aging and in Alzheimer's disease. Lipids 26 421-425. doi:10.1007/BF02536067

Sparks, R. P., Guida, W. C., Sowden, M. P., Jenkins, J. L., Starr, M. L., Fratti, R. A., Sparks, C. E. and Sparks, J. D. (2016). Sortilin facilitates VLDLB100 secretion by insulin sensitive McArdle RH7777 cells. Biochem. Biophys. Res. Commun. 478, 546-552. doi:10.1016/j.bbrc.2016.07.096

Strong, A., Ding, Q., Edmondson, A. C., Millar, J. S., Sachs, K. V., Li, X., Kumaravel, A., Wang, M. Y., Ai, D., Guo, L. et al. (2012). Hepatic sortilin regulates both apolipoprotein B secretion and LDL catabolism. J. Clin. Invest. 122 2807-2816. doi:10.1172/JCl63563

Subkhangulova, A., Malik, A. R., Hermey, G., Popp, O., Dittmar, G., Rathjen, T. Poy, M. N., Stumpf, A., Beed, P. S., Schmitz, D. et al. (2018). SORCS1 and SORCS3 control energy balance and orexigenic peptide production. EMBO Rep. 19, e44810. doi:10.15252/embr.201744810

Tripathi, S., Kushwaha, R., Mishra, J., Gupta, M. K., Kumar, H., Sanyal, S., Singh, D., Sanyal, S., Sahasrabuddhe, A. A., Kamthan, M. et al. (2017) Docosahexaenoic acid up-regulates both PI3K/AKT-dependent FABP7-PPAR interaction and MKP3 that enhance GFAP in developing rat brain astrocytes. J. Neurochem. 140, 96-113. doi:10.1111/jnc.13879

Vaegter, C. B., Jansen, P., Fjorback, A. W., Glerup, S., Skeldal, S., Kjolby, M., Richner, M., Erdmann, B., Nyengaard, J. R., Tessarollo, L. et al. (2011). Sortilin associates with Trk receptors to enhance anterograde transport and neurotrophin signaling. Nat. Neurosci. 14, 54-61. doi:10.1038/nn.2689

Xu, L. Z., Sánchez, R., Sali, A. and Heintz, N. (1996). Ligand specificity of brain lipid-binding protein. J. Biol. Chem. 271, 24711-24719. doi:10.1074/jbc.271.40. 24711

Yuki, D., Sugiura, Y., Zaima, N., Akatsu, H., Takei, S., Yao, I., Maesako, M., Kinoshita, A., Yamamoto, T., Kon, R. et al. (2014). DHA-PC and PSD-95 decrease after loss of synaptophysin and before neuronal loss in patients with Alzheimer's disease. Sci. Rep. 4, 7130. doi:10.1038/srep07130

Zhao, J., Fu, Y., Yamazaki, Y., Ren, Y., Davis, M. D., Liu, C.-C., Lu, W., Wang, X. Chen, K., Cherukuri, Y. et al. (2020a). APOE4 exacerbates synapse loss and neurodegeneration in Alzheimer's disease patient iPSC-derived cerebral organoids. Nat. Commun. 11, 5540. doi:10.1038/s41467-020-19264-0

Zhao, N., Ren, Y., Yamazaki, Y., Qiao, W., Li, F., Felton, L. M. Mahmoudiandehkordi, S., Kueider-Paisley, A., Sonoustoun, B., Arnold, M. et al. (2020b). Alzheimer's risk factors age, APOE genotype, and sex drive distinct molecular pathways. Neuron 106, 727-742.e6. doi:10.1016/j.neuron.2020.02.034 